\title{
Exact solutions and critical chaos in dilaton gravity with a boundary
}

\author{
Maxim Fitkevich, ${ }^{a, b}$ Dmitry Levkov $^{a}$ and Yegor Zenkevich Z $^{c, d, e, 1}$ \\ ${ }^{a}$ Institute for Nuclear Research of the Russian Academy of Sciences, \\ 60th October Anniversary Prospect 7a, Moscow 117312, Russia \\ ${ }^{b}$ Moscow Institute of Physics and Technology, \\ Institutskii per. 9, Dolgoprudny 141700, Moscow Region, Russia \\ ${ }^{c}$ Dipartimento di Fisica, Università di Milano-Bicocca, \\ Piazza della Scienza 3, I-20126 Milano, Italy \\ ${ }^{d}$ INFN, sezione di Milano-Bicocca, \\ I-20126 Milano, Italy \\ e National Research Nuclear University MEPhI, \\ Moscow 115409, Russia \\ E-mail: fitkevich@phystech.edu, levkov@ms2.inr.ac.ru, \\ yegor.zenkevich@gmail.com
}

ABSTRACT: We consider $(1+1)$-dimensional dilaton gravity with a reflecting dynamical boundary. The boundary cuts off the region of strong coupling and makes our model causally similar to the spherically-symmetric sector of multidimensional gravity. We demonstrate that this model is exactly solvable at the classical level and possesses an on-shell $\mathrm{SL}(2, \mathbb{R})$ symmetry. After introducing general classical solution of the model, we study a large subset of soliton solutions. The latter describe reflection of matter waves off the boundary at low energies and formation of black holes at energies above critical. They can be related to the eigenstates of the auxiliary integrable system, the Gaudin spin chain. We argue that despite being exactly solvable, the model in the critical regime, i.e. at the verge of black hole formation, displays dynamical instabilities specific to chaotic systems. We believe that this model will be useful for studying black holes and gravitational scattering.

KEYwords: 2D Gravity, Black Holes, Integrable Field Theories

ARXIV EPRINT: 1702.02576

\footnotetext{
${ }^{1}$ On leave of absence from ITEP, Moscow 117218, Russia.
} 


\section{Contents}

1 Introduction 1

2 The model 4

2.1 Adding the boundary 4

2.2 Solution in the bulk and reflection laws 5

$\begin{array}{lll}2.3 & \text { Simple equation for the boundary } & 7\end{array}$

2.4 On-shell conformal symmetry 9

3 Integrable sector $\quad 11$

3.1 General solution $\quad 11$

$\begin{array}{lll}3.2 & \text { Soliton solutions with power-law singularities } & 11\end{array}$

$\begin{array}{lll}3.3 & \text { Simplifying the coefficient equations } & 14\end{array}$

$\begin{array}{lll}3.4 & \mathrm{SL}(2, \mathbb{C}) \text { symmetry } & 14\end{array}$

$\begin{array}{ll}3.5 & \text { Relation to the Gaudin model } \\ 3.6 & \text { Positivity }\end{array}$

$\begin{array}{ll}3.6 & \text { Positivity condition } \\ \end{array}$

$\begin{array}{llr}4 & \text { Critical chaos } & 19\end{array}$

$\begin{array}{ll}\text { 4.1 Perturbative expansion in the critical regime } & 19\end{array}$

$\begin{array}{lll}4.2 & \text { Shock-wave instability } & 21\end{array}$

5 Discussion 23

A Field equations and boundary conditions $\quad 23$

$\begin{array}{lll}\text { A.1 Derivation } & 23\end{array}$

$\begin{array}{ll}\text { A.2 Solution in the conformal gauge } & 24\end{array}$

B Bethe Ansatz for the Gaudin model 25

\section{Introduction}

The models of two-dimensional dilaton gravity were popular for decades [1-3]. Some of them describe spherically-symmetric sectors of multidimensional gravities with dilaton fields $\phi$ related to the sizes of the extra spheres. ${ }^{1}$ Some others are exactly solvable at the semiclassical $[4,5]$ or quantum [3] levels which makes them valuable for studying black holes and gravitational scattering [6-8].

These models become particularly important in the context of information paradox $[9,10]$ confronting an apparent loss of quantum coherence during black hole evaporation

\footnotetext{
${ }^{1}$ In particular, gravitational sector of the CGHS model [4] can be obtained by spherical reduction of $D$-dimensional gravity at $D \rightarrow+\infty[3]$.
} 


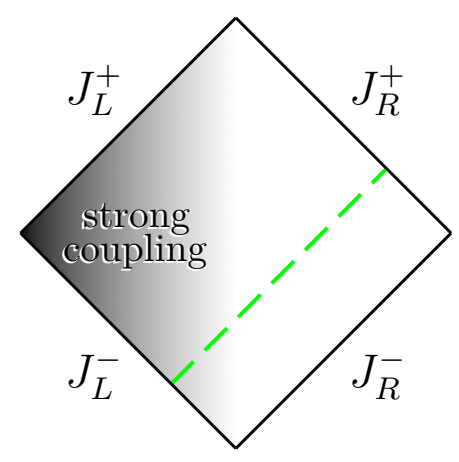

(a)

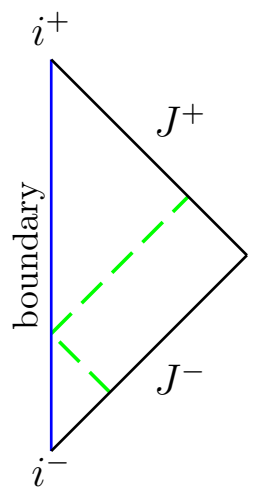

(b)

Figure 1. Penrose diagrams of Minkowski vacuum in the (a) original CGHS model and (b) model with a boundary. The dashed lines are light rays extending from $J^{-}$to $J^{+}$.

with the principles of quantum theory. Since unitarity of quantum gravity is strongly supported by the AdS/CFT correspondence [11, 12], modern AMPS argument [13, 14] suggests dramatic violation of the equivalence principle ("firewalls") in the vicinity of old black hole horizons, see $[15,16]$ for earlier works. This feature, if exists, may leave "echoes" in the gravitational wave signal $[17,18]$ to be detected by LIGO $[19,20]$, cf. [21, 22]. From the theoretical viewpoint, further progress can be achieved by understanding unitary evolution of black holes outside of the explicit AdS/CFT framework. This brings us to the arena of two-dimensional models which may, in addition, clarify relation of black holes to quantum chaos [23-28], cf. [29].

Unfortunately, solvable models of two-dimensional dilaton gravity essentially differ from their multidimensional cousins. Consider e.g. the celebrated Callan-Giddings-HarveyStrominger (CGHS) model [4], see [1, 2] for reviews. Its two-dimensional Minkowski vacuum in figure 1a, unlike the multidimensional vacua, has disconnected sets of "left" and "right" infinities $J_{L}^{ \pm}$and $J_{R}^{ \pm}$, and transitions between those are expected [30] to be important for the information loss problem. Besides, the CGHS model is strongly coupled [31] near the "left" infinities which puts its semiclassical results on shaky ground. It was recently suggested [32] that due to the above peculiarities evaporation of the CGHS black holes leads to remnants rather than firewalls.

We consider the modified CGHS model proposed ${ }^{2}$ in [33, 34], see also [31, 39-42]. The region of strong coupling in this model is cut off by the reflective dynamical boundary placed at a fixed value $\phi=\phi_{0}$ of the dilaton field, see figure $1 \mathrm{~b}$. Parameter $\mathrm{e}^{2 \phi_{0}} \ll 1$ plays the role of a small coupling constant. We explicitly obtain reparametrization-invariant action of the model by restricting CGHS action to the space-time region $\phi<\phi_{0}$ and adding appropriate boundary terms. Note that the original CGHS model is formally restored in the limit $\phi_{0} \rightarrow+\infty$ which shifts the regulating boundary in figure $1 \mathrm{~b}$ all the way the left. We do not consider this limit avoiding potential problems with strong coupling, cf. [43-45].

\footnotetext{
${ }^{2}$ Similar models appeared recently in the context of near $\mathrm{AdS}_{2} /$ near $\mathrm{CFT}_{1}$ holography [35-38].
} 


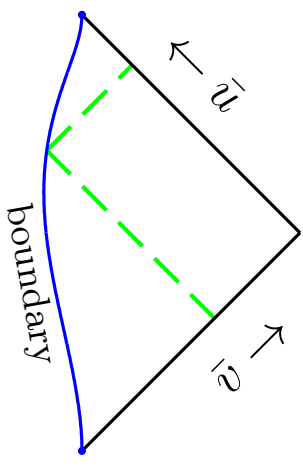

(a) low-energy

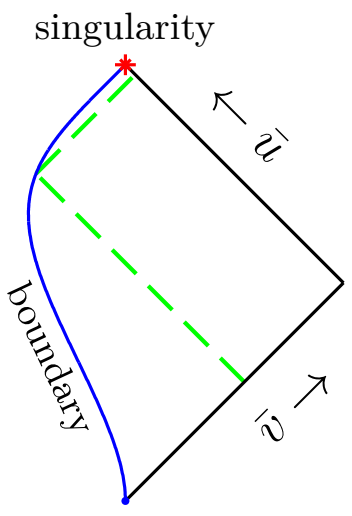

(b) critical

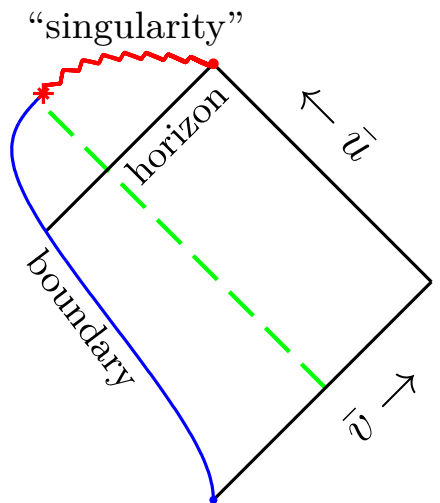

(c) high-energy

Figure 2. The simplest exact solution $\left(s_{1}=s_{2}=1\right)$ in the model with a boundary at different values of parameters. Finite-range light-cone coordinates $(\bar{u}, \bar{v})$ are used. The centers of the incoming and reflected matter wave packets are marked by the dashed lines.

As an additional bonus, the above model with a boundary is causally similar to spherically-symmetric multidimensional gravity, cf. figure $1 \mathrm{~b}$. The price to pay, however, is nonlinear equation of motion for the boundary which, if non-integrable, may damage major attractive property of the CGHS model - its solvability. Note that the previous studies of this or similar models were relying on numerical $[40-42,46]$ or shock-wave $[33,34,39]$ solutions.

In this paper we demonstrate that the CGHS model with a boundary is exactly solvable at the classical level. We obtain general solution of the classical field equations and construct an infinite number of particular soliton solutions. The latter describe reflection of matter waves off the boundary at low energies and formation of black holes at energies above some critical values, see figures $2 \mathrm{a}$ and 2 c. Each solution is characterized by $N$ integers or half-integers $s_{1}, \ldots, s_{N}$ and the same number of real parameters. The parameters of the solitons satisfy inequalities ensuring positivity of energy.

We establish one-to-one correspondence between the above solitons and the eigenstates of the auxiliary integrable system - the rational Gaudin model [47-49]. This allows us to classify these solutions and study their properties.

We find that equation of motion for the boundary is invariant under conformal transformations $v \rightarrow w(v)$, where $v$ is the light-cone coordinate, $w(v)$ is an arbitrary function. These transformations relate physically distinct solutions, and one should not confuse them, say, with the residual reparametrization symmetry in $[39,40]$. In particular, the transformations from the global $\mathrm{SL}(2, \mathbb{R})$ subgroup change massless matter field(s) $f$ of the model as the standard zero-weight fields. They also map the solitons into solitons. The transformations with nonzero Schwarzian derivative act non-linearly on $f$, and we do not consider them in detail.

Finally, we study dynamics of the model in the critical regime, i.e. at the verge of black hole formation, cf. figure $2 \mathrm{~b}$. We demonstrate that in this limit scattering of matter 
waves off the boundary displays instabilities specific to chaotic systems: the final state of the process becomes extremely sensitive to the initial Cauchy data. This feature is in tune with the near-horizon chaos suggested in [25]. We argue that it impedes global integrability of the model, i.e. prevents one from choosing a complete set of smooth conserved quantities in the entire phase space.

In section 2 we introduce dilaton gravity with a boundary and study its properties. We construct exact solutions in section 3. Critical chaos is considered in section 4. In section 5 we discuss possible applications of our results.

\section{The model}

\subsection{Adding the boundary}

We consider two-dimensional model with classical action

$$
\begin{aligned}
S= & \int_{\phi<\phi_{0}} d^{2} x \sqrt{-g}\left[e^{-2 \phi}\left(R+4(\nabla \phi)^{2}+4 \lambda^{2}\right)-(\nabla f)^{2} / 2\right] \\
& +\int_{\phi=\phi_{0}} d \tau e^{-2 \phi}(2 K+4 \lambda)
\end{aligned}
$$

where $^{3}$ the integrand in the first line is the CGHS Lagrangian [4] describing interaction of the metric $g_{\mu \nu}$ and dilaton $\phi$ with massless scalar $f$; the dimensionful parameter $\lambda$ sets the energy scale of the model. In eq. (2.1) we modified the CGHS action by restricting integration to the submanifold $\phi<\phi_{0}$ and adding the boundary terms ${ }^{4}$ at $\phi=\phi_{0}$. We introduced the proper time of the boundary $\tau$, its extrinsic curvature $K=g^{\mu \nu} \nabla_{\mu} n_{\nu}$, and unit outer normal $n_{\mu} \propto \nabla_{\mu} \phi$.

In fact, the choice of the boundary action in eq. (2.1) is limited. First, the GibbonsHawking term with extrinsic curvature ensures consistency of the gravitational action. Without this term the boundary conditions following from eq. (2.1) would be incompatible with the Dirichlet condition $\phi=\phi_{0}$, see [51] and cf. appendix A.1. Second, we assume no direct interaction of the matter field $f$ with the boundary. Then the only natural generalization of our model would include an arbitrary constant in the last term of eq. (2.1). However, this parameter needs to be fine-tuned in order to retain Minkowski solution (see below). Thus, the action (2.1) describing interaction of the boundary with the gravitational sector of the CGHS model is fixed [33].

The quantity $\mathrm{e}^{2 \phi_{0}}$ is a coupling constant controlling loop expansion in the model (2.1). Indeed, change of variables $\tilde{\phi}=\phi-\phi_{0}, \tilde{f}=\mathrm{e}^{\phi_{0}} f$ brings this parameter in front of the classical action, $S=\tilde{S} / \mathrm{e}^{2 \phi_{0}}$. Thus, $\mathrm{e}^{2 \phi_{0}}$ plays the role of a Planck constant implying that the model is classical at $\mathrm{e}^{2 \phi_{0}} \ll 1$.

It is clear that the bulk equations in the model (2.1) are the same as in the original CGHS model $[1,2,4]$. However, extremizing the action with respect to the boundary values

\footnotetext{
${ }^{3}$ We use $(-,+)$ signature and Greek indices $\mu, \nu, \cdots=0,1$. We denote covariant derivatives by $\nabla_{\mu}$ and Ricci scalar by $R$.

${ }^{4}$ Similar boundary terms appear in the path integral formulation of dilaton gravity [50].
} 
of $g_{\mu \nu}$ and $f$, one also obtains the boundary conditions

$$
n^{\mu} \nabla_{\mu} \phi=\lambda, \quad n^{\mu} \nabla_{\mu} f=0 \quad \text { at } \quad \phi=\phi_{0},
$$

see appendix A.1 for details. Note that the constant $\lambda$ in the right-hand side of the first equation comes from the last term in eq. (2.1). Besides, the second equation guarantees zero energy flux through the boundary.

Let us now recall [4] that linear dilaton vacuum

$$
g_{\mu \nu}=\eta_{\mu \nu}, \quad \phi=-\lambda x, \quad f=0,
$$

satisfies the CGHS equations, cf. appendix A.1. In this case the boundary $\phi=\phi_{0}$ is static, $x_{\text {boundary }}=-\phi_{0} / \lambda$, and the first of eqs. (2.2) is automatically satisfied. Note that the Minkowski vacuum (2.3) is a solution in our model due to exact matching between the bulk and boundary terms with $\lambda$ in the action (2.1).

\subsection{Solution in the bulk and reflection laws}

The CGHS equations in the bulk are exactly solvable $[1,2]$ in the light-cone frame $(u, v)$, where

$$
d s^{2}=-e^{2 \rho(u, v)} d u d v .
$$

Let us review their general solution leaving technical details to appendix A.2. In what follows we fix the remaining gauge freedom in eq. (2.4) with the on-shell "Kruskal" condition $\rho=\phi$.

In the frame (2.4) the matter field satisfies $\partial_{u} \partial_{v} f=0$ and therefore splits into a sum of incoming and outgoing parts,

$$
f=f_{\text {in }}(v)+f_{\text {out }}(u)
$$

The respective energy fluxes are

$$
T_{v v}(v)=\left(\partial_{v} f_{\text {in }}\right)^{2} \quad \text { and } \quad T_{u u}(u)=\left(\partial_{u} f_{\text {out }}\right)^{2} .
$$

This specifies the Cauchy problem in our model: one prepares $f_{\text {in }}$ or $T_{v v}$ at the past null infinity and calculates $f_{\text {out }}$ or $T_{u u}$ at $J^{+}$, see figure $1 \mathrm{~b}$.

The solution for the scale factor $\rho$ and dilaton field $\phi$ is

$$
e^{-2 \rho}=e^{-2 \phi}=-\lambda^{2} v u+g(v)+h(u),
$$

where

$$
g(v)=\frac{1}{2} \int_{0}^{v} d v^{\prime} \int_{v^{\prime}}^{+\infty} d v^{\prime \prime} T_{v v}\left(v^{\prime \prime}\right), \quad h(u)=-\frac{1}{2} \int_{-\infty}^{u} d u^{\prime} \int_{-\infty}^{u^{\prime}} d u^{\prime \prime} T_{u u}\left(u^{\prime \prime}\right) .
$$

We fixed the integration constants in these expressions by requiring, first, that the spacetime is flat in the infinite past, i.e. no white hole preexists the scattering process. Second, we chose the coordinates in such a way that the quadrant $u \in(-\infty ; 0), v \in(0 ;+\infty)$ covers 


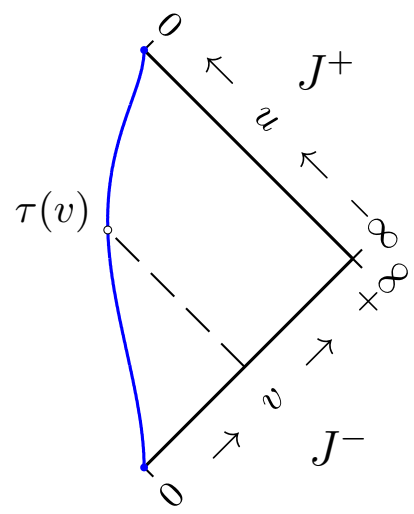

Figure 3. Penrose diagram showing the ranges of $u, v$ and definition of $\tau(v)$.

all space-time accessible to the distant observer. In particular, the limits $u \rightarrow-\infty$ at $v>0$ and $v \rightarrow+\infty$ at $u<0$ lead to $J^{-}$and $J^{+}$, respectively, see figure 3 .

Now, consider the boundary $\phi=\phi_{0}$ described by the function $u=U(v)$ in the "Kruskal" coordinates. Substituting the bulk solution (2.5), (2.7) into the boundary conditions (2.2), one obtains equation for $U(v)$ and reflection law for the matter field $f$,

$$
\frac{d U}{d v}=\frac{e^{2 \phi_{0}}}{\lambda^{2}}\left(\partial_{v} g-\lambda^{2} U\right)^{2}, \quad f_{\text {out }}(U(v))=f_{\text {in }}(v)
$$

see appendix A.2 for the derivation of these equations and proof that they are compatible with the definition $\phi(U(v), v)=\phi_{0}$ of the boundary. Note that the second of eqs. (2.9) relates the incoming and outgoing waves by conformal transformation $v \rightarrow U(v)$. The first equation implies that the boundary is always time-like, $d U / d v>0$. When rewritten in the appropriate terms, it coincides ${ }^{5}$ with the boundary equation obtained in [33, 34, 39] using energy conservation.

One easily finds solution in the empty space using eqs. (2.9) and (2.7) with $T_{v v}=$ $T_{u u}=0$,

$$
U(v)=-\mathrm{e}^{-2 \phi_{0}} /\left(\lambda^{2} v\right), \quad \mathrm{e}^{-2 \rho}=\mathrm{e}^{-2 \phi}=-\lambda^{2} u v, \quad f=0,
$$

where the integration constant in the first expression was chosen to make $U(v)$ smooth and invertible in the interval $0<v<+\infty$. Solution (2.10) is the linear dilaton vacuum: ${ }^{6}$ coordinate transformation

$$
\lambda v=e^{\lambda(t+x)}, \quad \lambda u=-e^{-\lambda(t-x)}
$$

brings it to the standard form (2.3). In what follows we impose flat asymptotics (2.10) in the infinite past $v \rightarrow 0, u \rightarrow-\infty$.

Note that the space-time (2.7) is always flat far away from the boundary, i.e. at large $|u|$ and $v$. Below we transform to the asymptotic Minkowski coordinates $(t, x)$ using eq. (2.11).

\footnotetext{
${ }^{5}$ It does not conform, however, with the boundary conditions introduced at one-loop level in [43-45]: in the classical model the latter conditions imply that the boundary is space-like.

${ }^{6}$ Recall that we excluded solutions with eternal black holes in eq. (2.7).
} 
We have got a receipt for solving the Cauchy problem in the CGHS model with a boundary. In this case the initial Cauchy data are represented by the incoming wave $f_{\text {in }}(v)$ or its energy flux $T_{v v}(v)$. One solves eqs. (2.9) with the initial condition (2.10) at $v \rightarrow 0$ and finds $U(v), f_{\text {out }}(u)$. The scale factor of the metric, dilaton and matter fields are then given by eqs. (2.7) and (2.5).

\subsection{Simple equation for the boundary}

One notices that eq. (2.9) for $U(v)$ is, in fact, a Riccati equation. The standard substitution

$$
\lambda^{2} U=\partial_{v} g-e^{-2 \phi_{0}} \partial_{v} \psi / \psi,
$$

brings it to the form of a Schrödinger equation for the new unknown $\psi(v)$,

$$
\partial_{v}^{2} \psi(v)=-\frac{\mathrm{e}^{2 \phi_{0}}}{2} T_{v v}(v) \psi(v)
$$

Note that $\psi(v)$ is defined up to a multiplicative constant. Now, one can solve for $\psi(v)$ given the initial data $T_{v v}(v)$. After that the entire solution is determined by eq. (2.12) and expressions from the previous section. For example, the outgoing energy flux equals

$$
T_{u u}(u)=\left.\left(\lambda e^{\phi_{0}} \psi / \partial_{v} \psi\right)^{4} T_{v v}\right|_{v=V(u)},
$$

where $V(u)$ is inverse of $U(v), V(U(v))=v$. We obtained eq. (2.14) by substituting the reflection law (2.9) into the definition (2.6) of the flux and then expressing the derivative of $U(v)$ from the first of eqs. (2.9) and eq. (2.12).

Importantly, eq. (2.13) is well-known in mathematical physics. Similar equation appears in Liouville theory at classical and semiclassical levels [52]. Besides, the eigenstates of the Gaudin model [47] can be related to the solutions of eq. (2.13) with monodromies \pm 1 and rational $T_{v v}(v)$ [48]. In what follows we exploit these similarities for studying exact solutions in dilaton gravity.

The function $\psi(v)$ in eq. (2.12) has simple geometric meaning. First, the value of $\psi$ is related to the proper time $\tau$ along the boundary,

$$
d \tau^{2}=e^{2 \phi_{0}} d U(v) d v=\left(\partial_{v} \psi / \lambda \psi\right)^{2} d v^{2} \quad \Rightarrow \quad \psi(v)=\psi_{0} \cdot \mathrm{e}^{\lambda \tau(v)},
$$

where we used eqs. (2.4), (2.9), (2.12) and introduced the arbitrary constant $\psi_{0}$ related to the origin of $\tau$. Function $\tau(v)$ is illustrated in figure 3. Second, recall that $v$ is the exponent of the flat light-cone coordinate $(t+u)$ far away from the boundary, eq. (2.11). Thus, $\psi(v)$ maps the affine coordinate at $J^{-}$to $\tau$. Equation (2.13) relates this coordinate-independent function to the asymptotic Cauchy data $T_{v v}(v)$.

Consider general properties of classical solutions in the model with a boundary. Expression (2.15) implies that $\psi(v)$ vanishes in the infinite past,

$$
\psi(0)=0 \text {. }
$$




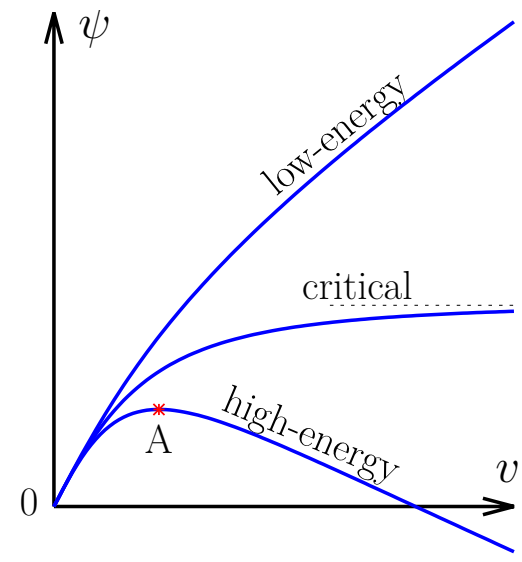

(a)

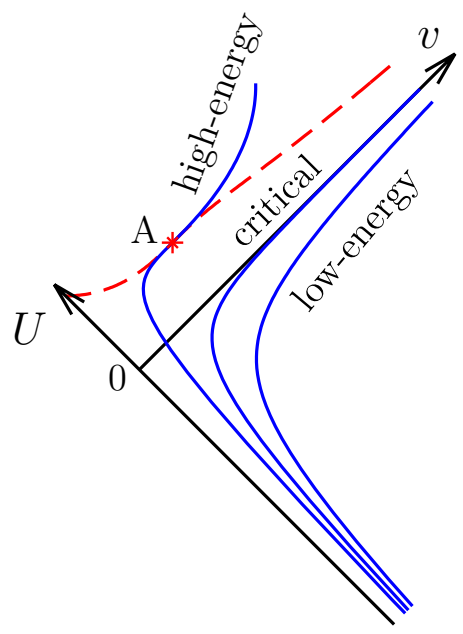

(b)

Figure 4. Functions $\psi(v)$ and $U(v)$ at different $T_{v v}$. The right graph is rotated for visualization purposes. Dashed line in this graph is the space-like "singularity" $\phi=\phi_{0}$.

Indeed, behavior $\psi \rightarrow c_{0} v$ as $v \rightarrow 0$ corresponds to the linear dilaton vacuum (2.10) in the beginning of the process. To simplify the next argument, we $\operatorname{set}^{7} c_{0}=1$. We consider well-localized $T_{v v}(v)$ and therefore linear asymptotics

$$
\psi(v) \rightarrow C v+D \quad \text { as } \quad v \rightarrow+\infty
$$

of the solution to eq. (2.13). If $T_{v v}$ is small, one has $C \approx 1$. The respective "low-energy" solutions describe reflection of matter waves off the time-like boundary, see figures 4a,b. As $T_{v v}$ grows, the function $\psi(v)$ becomes more concave and $C$ decreases because $\partial_{v}^{2} \psi \propto$ $-T_{v v}<0$. For some large fine-tuned $T_{v v}(v)$ one obtains critical solutions with $C=0$. In this case the boundary is null in the asymptotic future because its proper time $\tau(v)$ in eq. (2.15) remains finite as $v \rightarrow+\infty$. The respective "critical" solution in figures 4 is at the brink of black hole formation: we will see that the asymptotically null boundary sits precisely at the horizon of would-be black hole.

At sufficiently high energies we get $C<0$ and therefore $\psi(v)$ has a maximum (point $A$ in figure 4a). The boundary is null at this point: $d U /\left.\left.d v\right|_{A} \propto\left(\partial_{v} \psi\right)^{2}\right|_{A}=0$ according to eqs. (2.9) and (2.12). Moreover, near $A=\left(u_{A}, v_{A}\right)$ one obtains $U(v) \approx u_{A}+d \cdot\left(v-v_{A}\right)^{3}$ and

$$
\mathrm{e}^{-2 \phi(u, v)} \approx \mathrm{e}^{-2 \phi_{0}}+\frac{T_{v v}\left(v_{A}\right)}{4 d^{2 / 3}}\left[\left(u_{A}-u\right)^{2 / 3}-d^{2 / 3}\left(v-v_{A}\right)^{2}\right] .
$$

where eqs. (2.9), (2.12), and (2.7) were solved to the leading order in $u-u_{A}, v-v_{A}$. Thus, $A$ is a singularity of $\phi$ in coordinates $(u, v)$.

Besides, one discovers that the condition $\phi=\phi_{0}$ defines two intersecting curves $u-$ $u_{A} \approx \pm d\left(v-v_{A}\right)^{3}$ near $A$, and only one of those is the time-like boundary considered

\footnotetext{
${ }^{7}$ Recall that $\psi(v)$ is defined up to a multiplicative constant.
} 
so far. The second curve is space-like, it is shown by the dashed line in figure $4 \mathrm{~b}$. The boundary conditions (2.9) are not met at this line. We obtained the analog of the black hole singularity in the model with a boundary. Indeed, our model is formulated at $\phi<\phi_{0}$ i.e. in the space-time region to the right of both solid and dashed graphs in figure 4b. The space-like "edge" $\phi=\phi_{0}$ swallows all matter at $u>0$ limiting the region accessible to the outside observer to $u<0$. The line $u=0$ is a horizon.

Except for the point $A$ itself, the solution is smooth at the space-like "singularity" $\phi=\phi_{0}$. This fact was not appreciated in the previous studies. The mass of the formed black hole, by energy conservation, is related to the value of the dilaton field at the future horizon,

$$
M_{b h}=\int_{0}^{+\infty} \lambda v d v T_{v v}-\int_{-\infty}^{0} \lambda|u| d u T_{u u}=2 \lambda[g(+\infty)+h(0)]=2 \lambda \lim _{v \rightarrow+\infty} \mathrm{e}^{-2 \phi(0, v)},
$$

where we subtracted the final matter energy from the initial one in the first equality (cf. eq. (2.11)), integrated by parts and used eqs. (2.8) in the second equality, and then expressed the result in terms of $\phi$, eq. (2.7). Since $\phi<\phi_{0}$, this implies that all black hole masses are larger than

$$
M_{c r}=2 \lambda \mathrm{e}^{-2 \phi_{0}},
$$

see detailed discussion in [5, 42]. Black holes with $M_{b h}=M_{c r}$ have boundary sitting precisely at the horizon. They are formed in the critical solutions.

The solutions in figure $4 \mathrm{~b}$, when replotted in the finite-range coordinates $(\bar{u}, \bar{v})=$ $(\arctan u, \arctan v)$, look like Penrose diagrams, see figure 2. From now on, we will exploit $\bar{u}$ and $\bar{v}$ for visualizing the solutions. We will also mark the (smooth) space-like "singularities" $\phi=\phi_{0}$ by zigzag lines, see the one in figure 2c.

\subsection{On-shell conformal symmetry}

We find that the boundary equation (2.13) is invariant under conformal transformations $v \rightarrow w(v)$,

$$
\begin{aligned}
\psi \rightarrow \tilde{\psi}(w) & =\left(\frac{d v}{d w}\right)^{-1 / 2} \psi(v), \\
T_{v v} \mapsto \tilde{T}_{v v}(w) & =\left(\frac{d v}{d w}\right)^{2} T_{v v}(v)+\mathrm{e}^{-2 \phi_{0}}\{v ; w\},
\end{aligned}
$$

which change $\psi(v)$ as an $h=-1 / 2$ primary field and $T_{v v}(v)$ as an energy-momentum tensor with large negative central charge $c=-24 \pi \mathrm{e}^{-2 \phi_{0}}$ [53]. In eq. (2.19) we introduced the Schwarzian derivative $\{v ; w\} \equiv v^{\prime \prime \prime} / v^{\prime}-3\left(v^{\prime \prime}\right)^{2} / 2\left(v^{\prime}\right)^{2}$ with $v^{\prime} \equiv d v / d w$. The transformations (2.19), (2.20) relate physically distinct solutions ${ }^{8}$ with different energy fluxes $T_{v v}$. Acting with them on the vacuum $\psi=v, T_{v v}=0$ one can obtain any solution.

\footnotetext{
${ }^{8}$ Unlike the transformations in [39, 40], they do not represent residual gauge symmetry. The latter was completely fixed, see discussion after eq. (2.4).
} 
Note that the symmetry (2.19), (2.20) does not make our model a CFT in a conventional sense. ${ }^{9}$ First, the full energy-momentum tensor $T_{\mu \nu}+T_{\mu \nu}^{(\phi)}$ of the model includes contribution of the dilaton field and vanishes by Einstein equations, cf. eq. (A.1). Second, eq. (2.20) is not a conformal transformation $f_{\text {in }} \rightarrow f_{\text {in }}(v(w))$ of the massless scalar field $f$ far away from the boundary: the latter changes classical $T_{v v} \equiv\left(\partial_{v} f_{\text {in }}\right)^{2}$ without the Schwarzian derivative. At the quantum level, healthy conformal matter has positive central charge $c>0$ [53], and transformations of its energy-momentum tensor $T_{v v}$ do not match eq. (2.20) as well.

Transformations from the $\mathrm{SL}(2, \mathbb{R})$ subgroup of $(2.19),(2.20)$,

$$
v \rightarrow w(v)=\frac{\alpha v+\beta}{\gamma v+\delta}, \quad \alpha \delta-\beta \gamma=1,
$$

have vanishing Schwarzian derivative and therefore change $f$ in the standard way $f_{\text {in }} \rightarrow f_{\text {in }}(v(w))$. Besides trivial translations of $v$ they include $v$-dilatations due to shifts of the asymptotic coordinate $t+x$ in eq. (2.11) and inversion $v \rightarrow 1 / v$ related to PTreflection $t+x \rightarrow-(t+x)$. These transformations constitute the global symmetry group of our model.

As a side remark, let us argue that (2.19), (2.20) is a symmetry of the gravitational degrees of freedom but not of the matter sector. To this end we introduce the field $\chi(u)=$ $\mathrm{e}^{-\lambda \tau(u)} / \psi_{0}$ which is $T$-symmetric with respect to $\psi(v)$ and therefore satisfies

$$
\partial_{u}^{2} \chi(u)=-\frac{\mathrm{e}^{2 \phi_{0}}}{2} T_{u u}(u) \chi(u)
$$

cf. eqs. (2.15) and (2.13); now, $\tau(u)$ is the boundary proper time parametrized with $u$. It is convenient to combine $\psi(v)$ and $\chi(u)$ into a single free field

$$
\mathrm{e}^{-2 \Phi(u, v)} \equiv \chi(u) \psi(v) \mathrm{e}^{-2 \phi_{0}},
$$

transforming in a simple Liouville-like manner under eq. (2.19). To describe the gravitational degrees of freedom with $\Phi$, we extract its energy-momentum tensor $T_{\mu \nu}^{(\phi)}$ from the Einstein equations $T_{\mu \nu}^{(\phi)}+T_{\mu \nu}=0$,

$$
T_{v v}^{(\phi)} \equiv-T_{v v}=8 \mathrm{e}^{-2 \phi_{0}}\left[\left(\partial_{v} \Phi\right)^{2}-\partial_{v}^{2} \Phi / 2\right], \quad T_{u u}^{(\phi)} \equiv-T_{u u},
$$

where eq. (2.13) was used in the left equality; similar expression for $T_{u u}^{(\phi)}$ can be obtained using eq. (2.22). One observes that $T_{v v}^{(\phi)}$ transforms under eq. (2.19) as an energy-momentum tensor with positive conformal charge $c=24 \pi \mathrm{e}^{2 \phi_{0}}$, in agreement with eq. (2.20).

Now, the entire scattering problem can be reformulated in terms of $\Phi$. One sends the incoming energy flux $T_{v v}^{(\phi)}$ towards the dynamical boundary $u=U(v)$ at $\Phi=\phi_{0}$. The flux reflects into $T_{u u}^{(\phi)}$ according to the non-conformal law $T_{u u}^{(\phi)}=(d U / d v)^{-2} T_{v v}^{(\phi)}$, see eq. (2.14). All these equations and boundary conditions can be summarized in the flat-space action

$$
S_{\Phi}=-\int_{\Phi<\phi_{0}} d^{2} x\left[\mathrm{e}^{-2 \phi_{0}}\left(\partial_{\mu} \Phi\right)^{2}+\lambda^{2}\right]
$$

\footnotetext{
${ }^{9}$ Thus, one may still hope that our model is unitary at the quantum level despite negative primary dimension in eq. (2.19) and negative central charge in eq. (2.20).
} 
In this setup (2.19), (2.20) is an apparent conformal symmetry of $\Phi$ far away from the boundary, whereas the symmetry of the matter sector is hidden in the reflection laws.

\section{Integrable sector}

\subsection{General solution}

One can use eq. (2.13) to express the entire solution in terms of one arbitrary function. Indeed, introducing

$$
W \equiv \partial_{v} \psi / \psi=\mathrm{e}^{2 \phi_{0}}\left(\partial_{v} g-\lambda^{2} U\right)
$$

we find,

$$
\psi=e^{\int^{v} d v^{\prime} W\left(v^{\prime}\right)}, \quad-\frac{\mathrm{e}^{2 \phi_{0}}}{2} T_{v v}=W^{2}+\partial_{v} W .
$$

Then $U, T_{u u}, \phi$, and $f$ are given by eqs. (3.1), (2.14), (2.7), and (2.6). We obtained general classical solution in the model with a boundary.

By itself, this solution is of little practical use because the function $\psi(v)$ has a zero at $v=0$ and, possibly, another one at $v=\tilde{v}_{1}>0$, see figure $4 \mathrm{a}$. In general, the incoming flux $T_{v v}(v)$ in eq. (3.2) is singular at these points. Indeed, eq. (3.1) gives

$$
W(v)=R(v)+1 / v+1 /\left(v-\tilde{v}_{1}\right),
$$

where $R(v)$ is regular at $v \geq 0$. As a consequence, $T_{v v}(v)$ has first-order poles at $v=0$ and $\tilde{v}_{1}$. Requiring zero residuals at these poles, we obtain two constraints $R(0)=-R\left(\tilde{v}_{1}\right)=$ $1 / \tilde{v}_{1}$ on parameters of $R(v)$.

Choosing multiparametric $R(v)$ and solving the constraints, one finds an arbitrary number of smooth solutions. The physical ones satisfy

$$
T_{v v}(v) \geq 0, \quad \text { at } \quad v \geq 0 .
$$

In what follows we will concentrate on a large class of soliton solutions with power-law singularities. We will argue that some of them satisfy eq. (3.3).

\subsection{Soliton solutions with power-law singularities}

Let us follow the Painlevé test [54] and guess the form of $T_{v v}(v)$ which guarantees that the general solution $\psi(v)$ of eq. (2.13) has power-law singularities $\psi \sim\left(v-v_{0}\right)^{-s}$ in the complex $v$-plane. One introduces Laurent series at $v \approx v_{0}$,

$$
-\frac{\mathrm{e}^{2 \phi_{0}}}{2} T_{v v}=\sum_{k=0}^{+\infty} T_{k-2}\left(v-v_{0}\right)^{k-2}, \quad \psi=\sum_{k=0}^{+\infty} \psi_{k-s}\left(v-v_{0}\right)^{k-s}
$$

where the expansion of $T_{v v}$ starts from $\left(v-v_{0}\right)^{-2}$ due to eq. (2.13). Substituting eqs. (3.4) into eq. (2.13), we obtain an infinite algebraic system for $\psi_{k-s}$,

$$
(k-s)(k-s-1) \psi_{k-s}=T_{-2} \psi_{k-s}+T_{-1} \psi_{k-s-1}+\cdots+T_{k-2} \psi_{-s} .
$$




\begin{tabular}{|l|l|}
\hline$s$ & equation \\
\hline 1 & $T_{1}=T_{0} T_{-1}-\frac{1}{4}\left(T_{-1}\right)^{3}$ \\
$3 / 2$ & $T_{2}=\frac{2}{3} T_{1} T_{-1}-\frac{5}{18} T_{0}\left(T_{-1}\right)^{2}+\frac{1}{4}\left(T_{0}\right)^{2}+\frac{1}{36}\left(T_{-1}\right)^{4}$ \\
2 & $\ldots$ \\
\hline
\end{tabular}

Table 1. Equations for the Laurent coefficients of the solitonic $T_{v v}(v)$.

The very first $(k=0)$ of these relations gives $T_{-2}=s(s+1)$, the others determine $\psi_{k-s}$ with $k \geq 1$ in terms of arbitrary $\psi_{-s}$ and $\left\{T_{m}\right\}$. Expression (3.4) is a general solution of the second-order equation (2.13) if precisely two of its parameters, $\psi_{-s}$ and some $\psi_{k_{0}-s}$, remain arbitrary. Thus, $\left(k_{0}-s\right)\left(k_{0}-s-1\right)=s(s+1)$ in eq. (3.5) implying $k_{0}=2 s+1$. One concludes that $s$ is integer or half-integer.

Note that the two equations from the system (3.5) which do not determine the coefficients of $\psi$, constrain $\left\{T_{k}\right\}$. For example for $s=1 / 2$ one gets,

$$
T_{-2}=3 / 4, \quad T_{0}=\left(T_{-1}\right)^{2},
$$

where we expressed all $\psi_{k-1 / 2}$ via $\left\{T_{k}\right\}$ and $\psi_{-1 / 2}$. For larger $s$, one obtains $T_{-2}=s(s+1)$ and higher-order equations listed in table 1.

We arrived at the practical method for obtaining the soliton solutions in our model. One specifies $N$ singularities of $\psi(v)$ : selects their integer or half-integer powers $s_{n}$ and complex positions $v_{n}$. The function $T_{v v}(v)$ has second-order poles at $v=v_{n}$, see eq. (3.4). This analytic structure gives expressions,

$$
-\frac{\mathrm{e}^{2 \phi_{0}}}{2} T_{v v}=\sum_{n=1}^{N}\left[\frac{s_{n}\left(s_{n}+1\right)}{\left(v-v_{n}\right)^{2}}+\frac{T_{-1}^{n}}{v-v_{n}}\right], \quad \psi=C \frac{\prod_{m=1}^{M}\left(v-\tilde{v}_{m}\right)}{\prod_{n=1}^{N}\left(v-v_{n}\right)^{s_{n}}}
$$

where we required $T_{v v} \rightarrow 0$ as $v \rightarrow+\infty$ and introduced a polynomial in the nominator of $\psi(v)$ with $M$ zeroes $\tilde{v}_{m}$ and a normalization constant $C$. Next, one solves equations in table 1 at each singularity and determines $T_{-1}^{n}$. After that $\psi(v)$ is obtained by substituting eqs. (3.7) into eqs. (2.13) or (3.5). Two parameters - say, $C$ and $\tilde{v}_{M}-$ remain arbitrary because eq. (3.7) is a general solution of the second-order equation. One takes $\tilde{v}_{M}=0$ in accordance with the flat-space asymptotics (2.16). This gives the soliton $\left\{\psi(v), T_{v v}(v)\right\}$ characterized by $N$ complex parameters $v_{n}$ and the same number of integers or half-integers $s_{n}$.

We consider solutions with finite total energy of incoming matter,

$$
E_{\text {in }}=\int_{0}^{+\infty} \lambda v d v T_{v v}(v),
$$

see eq. (2.11). Convergence of this integral implies $T_{v v} \sim \bar{o}\left(v^{-2}\right)$ as $v \rightarrow+\infty$ or, given eq. (3.7), linear relations

$$
\sum_{n=1}^{N} T_{-1}^{n}=0, \quad \sum_{n=1}^{N}\left[s_{n}\left(s_{n}+1\right)+v_{n} T_{-1}^{n}\right]=0 .
$$




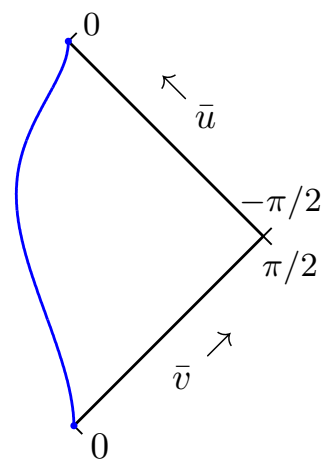

(a) $\lambda a=-0.2$

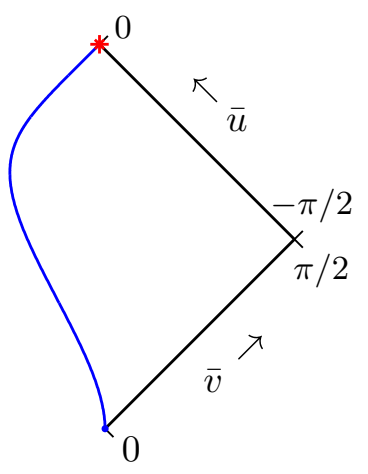

(b) $\lambda a=0$

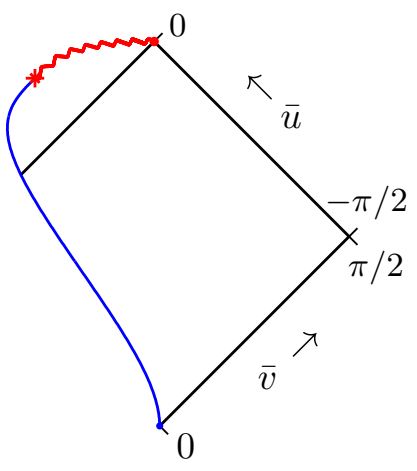

(c) $\lambda a=0.2$

Figure 5. Solution (3.10) in the finite-range coordinates $\bar{u}=\operatorname{arctg}(\lambda u), \bar{v}=\operatorname{arctg}(\lambda v)$ at different values of $a$. We use $\lambda b=\mathrm{e}^{2 \phi_{0}}=1$ keeping in mind that the parameter $\mathrm{e}^{2 \phi_{0}} \ll 1$ can be restored in the classical solution, see discussion in section 2.1 .

Moreover, asymptotic (2.17) of $\psi(v)$ suggests falloff $T_{v v} \sim O\left(v^{-4}\right)$ at large $v$ and additional relation

$$
\sum_{n=1}^{N}\left[2 v_{n} s_{n}\left(s_{n}+1\right)+v_{n}^{2} T_{-1}^{n}\right]=0,
$$

which should hold for noncritical solutions. Equations (3.8) and (3.9) are useful for obtaining the lowest solitons.

Example. Consider the soliton with two $s=1 / 2$ singularities. ${ }^{10}$ Solving the finite-energy conditions (3.8), one obtains $T_{-1}^{1}=-T_{-1}^{2}=3 /\left[2\left(v_{2}-v_{1}\right)\right]$. It is straightforward to check that $T_{v v}(v)$ with these parameters satisfies eqs. (3.6) at $v=v_{1}$ and $v=v_{2}$. To make the solution real at $v \in \mathbb{R}$, we take $v_{1}=a+i b$ and $v_{2}=a-i b$. Then eqs. (3.7) give,

$$
T_{v v}=\frac{6 \mathrm{e}^{-2 \phi_{0}} b^{2}}{\left[(v-a)^{2}+b^{2}\right]^{2}}, \quad \psi(v)=\frac{v\left(a^{2}+b^{2}-a v\right)}{\left[(v-a)^{2}+b^{2}\right]^{1 / 2}}
$$

where $\psi(v)$ was obtained by substituting eqs. (3.7) into eq. (2.13). One observes that the matter flux (3.10) peaks near $v \sim a$, its total energy $E_{\text {in }}=\frac{3}{2} M_{c r}\left[1+\frac{a}{b} \operatorname{arcctg}(-a / b)\right]$ is controlled by the ratio $a / b$, where $M_{c r}=2 \lambda \mathrm{e}^{-2 \phi_{0}}$ is the minimal black hole mass.

Since $\psi \rightarrow-a v$ as $v \rightarrow+\infty$, the solution (3.10) describes reflection of matter waves off the boundary and formation of black holes at $a<0$ and $a>0$, respectively, see figure 4a. This fact is clearly seen in figure 5 showing the boundary $u=U(v)$ at different $a$ in the finite-range coordinates $(\bar{u}, \bar{v})$. In figure $5 \mathrm{c}$ we also plotted the space-like "singularity" $\phi=\phi_{0}$ and horizon $u=0$ (zigzag red and solid black lines, respectively). Note that the critical solution in figure $5 \mathrm{~b}$ corresponds to $E_{\text {in }}=\frac{3}{2} M_{c r}$.

The simplest exact solution in eq. (3.10) describes the incoming matter flux with a single peak. Solutions with multiple peaks can be obtained by adding singularities at $v=a_{n} \pm i b_{n}$, see figure 6 . Unfortunately, it is hard to find these solutions explicitly at

\footnotetext{
${ }^{10}$ Note that $T_{v v}(v)$ with one singularity does not satisfy eqs. (3.8).
} 


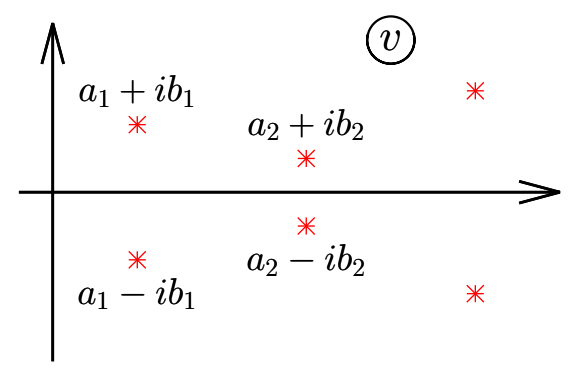

Figure 6. Singularities of solitons in the complex $v$-plane.

large $N$. Besides, it is not clear whether they will satisfy the positivity condition (3.3). We will clarify these issues in the subsequent sections.

\subsection{Simplifying the coefficient equations}

Instead of solving the equations in table 1 , one can extract $T_{v v}(v)$ from the general solution. Namely, substituting the solitonic $\psi(v)$ into the first of eqs. (3.2), we find,

$$
W(v)=-\sum_{n=1}^{N} \frac{s_{n}}{v-v_{n}}+\sum_{m=1}^{M} \frac{1}{v-\tilde{v}_{m}} .
$$

Then the second of eqs. (3.2) gives the incoming flux. However, in this case $T_{v v}(v)$ receives parasitic first-order poles at $v=\tilde{v}_{m}$ which are absent in eq. (3.7). Requiring zero residuals at these poles, we obtain equations for $\left\{\tilde{v}_{m}\right\}$,

$$
\sum_{n=1}^{N} \frac{s_{n}}{\tilde{v}_{m}-v_{n}}=\sum_{\substack{m^{\prime}=1 \\ m^{\prime} \neq m}}^{M} \frac{1}{\tilde{v}_{m}-\tilde{v}_{m^{\prime}}},
$$

which are, in fact, equivalent to the ones in table 1. Indeed, after solving eqs. (3.12) one obtains $T_{v v}(v)$ of the form (3.7) with

$$
T_{-1}^{n}=\sum_{n^{\prime} \neq n} \frac{2 s_{n} s_{n^{\prime}}}{v_{n}-v_{n^{\prime}}}-\sum_{m} \frac{2 s_{n}}{v_{n}-\tilde{v}_{m}} .
$$

In practice one finds $\tilde{v}_{m}$ numerically from eqs. (3.12), then computes $T_{v v}$ and $\psi$ by eqs. (3.13) and (3.7).

Unlike in section 3.1, we impose eqs. (3.12) at all $\tilde{v}_{m}$, not just the ones at the real positive axis. The goal is to obtain solutions with transparent properties, see the forthcoming discussion in section 3.5 .

\subsection{SL $(2, \mathbb{C})$ symmetry}

The global SL $(2, \mathbb{C})$ transformations $(2.21)$ are invertible and therefore preserve the singularity structure of the solitons. One obtains,

$$
T_{v v} \rightarrow \tilde{T}_{v v}(w)=\frac{T_{v v}(v)}{(\alpha-\gamma w)^{4}}, \quad \psi \rightarrow \tilde{\psi}(w)=(\alpha-\gamma w) \psi(v)
$$


This symmetry relates solitons with different parameters. Real solutions at $v \geq 0$ transform under $\operatorname{SL}(2, \mathbb{R})$.

The transformation (2.21) sends the point $v=-\delta / \gamma$ to infinity. If the original solution was regular at this point, its image receives asymptotics $\tilde{\psi} \rightarrow C w+D$ and $\tilde{T}_{v v} \rightarrow O\left(w^{-4}\right)$ as $w \rightarrow+\infty$. In eq. (2.17) we obtained the same asymptotics from physical considerations. Solutions with other asymptotics, i.e. those violating the finite-energy conditions (3.8) or eq. (3.9), have singularities "sitting" at infinity.

Example. One can use the above property to construct new solutions. Consider e.g. the trivial solution $\psi=v^{-s}-v^{s+1}, T_{v v}=-2 \mathrm{e}^{-2 \phi_{0}} s(s+1) / v^{2}$ of eq. (2.13) with non-linear $\psi(v)$ at large $v$. We send the points $v=0, \infty$, and 1 to $v_{1}, v_{2}$, and 0 by linear rational transformation $^{11}$ (3.14) and get,

$$
T_{v v}=\frac{-2 \mathrm{e}^{-2 \phi_{0}} s(s+1)\left(v_{2}-v_{1}\right)^{2}}{\left(v-v_{1}\right)^{2}\left(v-v_{2}\right)^{2}}, \quad \psi=\frac{i\left(v-v_{1}\right)^{s+1} v_{2}^{s}}{v_{1}^{s+1}\left(v-v_{2}\right)^{s}}-\frac{i\left(v-v_{2}\right)^{s+1} v_{1}^{s}}{v_{2}^{s+1}\left(v-v_{1}\right)^{s}}
$$

where the constant in front of $\psi(v)$ was ignored. This is the soliton with two singularities of power $s$. Taking $v_{1}=v_{2}^{*}=a+i b$, one obtains $T_{v v}(v) \geq 0$ at real $v$. Note that the incoming flux in eq. (3.15) is the same as in eq. (3.10) albeit with different multiplicative factor. The behaviors of the boundaries are also similar, as one can see by comparing the solutions (3.15) with ${ }^{12} s=1 / 2$ and 1 in figures 5 and 2 , respectively.

\subsection{Relation to the Gaudin model}

In this section we establish one-to-one correspondence between the solitons (3.7) and eigenstates of the auxiliary integrable system, the Gaudin model [47-49]. This will allow us to count the number of solitons and explain some of their properties.

The Gaudin model [47] describes a chain of $N$ three-dimensional spins $\hat{\boldsymbol{s}}_{n}=\left\{\hat{s}_{n}^{1}, \hat{s}_{n}^{2}, \hat{s}_{n}^{3}\right\}$ with the standard commutation relations $\left[\hat{s}_{n}^{\alpha}, \hat{s}_{l}^{\beta}\right]=i \delta_{n l} \epsilon^{\alpha \beta \gamma} \hat{s}_{n}^{\gamma}$. The model is equipped with $N$ commuting Hamiltonians

$$
\hat{\mathcal{T}}_{n}=\sum_{l \neq n} \frac{\left(\hat{\boldsymbol{s}}_{n}, \hat{\boldsymbol{s}}_{l}\right)}{v_{n}-v_{l}}
$$

where $v_{n}$ are complex parameters and $\left(\hat{\boldsymbol{s}}_{n}, \hat{\boldsymbol{s}}_{l}\right) \equiv \sum_{\alpha} \hat{s}_{n}^{\alpha} \hat{s}_{l}^{\alpha}$ is the scalar product. The eigenstates $|\Psi\rangle$ of the model simultaneously diagonalize all Hamiltonians, $\hat{\mathcal{T}}_{n}|\Psi\rangle=\mathcal{T}_{n}|\Psi\rangle$, where $\mathcal{T}_{n}$ are complex eigenvalues.

It is convenient to pack all spins and Hamiltonians into the operator-valued functions

$$
\hat{\boldsymbol{s}}(v) \equiv \sum_{n=1}^{N} \frac{\hat{\boldsymbol{s}}_{n}}{v-v_{n}}, \quad \hat{\mathcal{T}}(v) \equiv[\hat{\boldsymbol{s}}(v)]^{2}=\sum_{n=1}^{N}\left[\frac{\hat{\boldsymbol{s}}_{n}^{2}}{\left(v-v_{n}\right)^{2}}+\frac{2 \hat{\mathcal{T}}_{n}}{v-v_{n}}\right] .
$$

Now, the eigenvectors satisfy $\hat{\mathcal{T}}(v)|\Psi\rangle=\mathcal{T}(v)|\Psi\rangle$.

\footnotetext{
${ }^{11}$ With parameters $\alpha=-\beta=\left(1 / v_{2}-1 / v_{1}\right)^{-1 / 2}, \gamma=\alpha / v_{2}, \delta=\beta / v_{1}$.

${ }^{12}$ In figures $2 \mathrm{a}, \mathrm{b}$, and $\mathrm{c}$ we used $\lambda a=-1,-1 / \sqrt{3}$, and 0.3 , respectively, and $\lambda b=\mathrm{e}^{-2 \phi_{0}}=1$.
} 


\begin{tabular}{|c|l|l|}
\hline & Solitons & Eigenstates of the Gaudin model \\
\hline$v_{n}$ & positions of singularities & parameters of the Hamiltonians \\
$s_{n}$ & powers of singularities & representations of $\hat{\boldsymbol{s}}_{n}$ \\
$\tilde{v}_{m}$ & zeros of $\psi(v)$ & parameters of eigenstates \\
$T_{v v}=-2 \mathrm{e}^{-2 \phi_{0}} \mathcal{T}$ & incoming energy flux & eigenvalue of $\hat{\mathcal{T}}(v)$ \\
$T_{-1}^{n}=2 \mathcal{T}_{n}$ & coefficients of $T_{v v}$ & eigenvalues of $\hat{\mathcal{T}}_{n}$ \\
\hline
\end{tabular}

Table 2. Correspondence between solitons in dilaton gravity and eigenstates of the Gaudin model.

A complete set of eigenvectors and eigenvalues in the model (3.16) is provided by the algebraic Bethe Ansatz [47-49]. We review this method in appendix B and list its main results below.

One fixes the representations $\left(\hat{\boldsymbol{s}}_{n}\right)^{2}=s_{n}\left(s_{n}+1\right)$ of all spins, where $s_{n}$ are integers or half-integers. The simplest eigenstate $|0\rangle$ of the Gaudin model has all spins down,

$$
\hat{s}_{n}^{-}|0\rangle=0, \quad \hat{s}_{n}^{3}|0\rangle=-s_{n}|0\rangle \quad \text { for all } n,
$$

where $\hat{s}_{n}^{-} \equiv \hat{s}_{n}^{1}-i \hat{s}_{n}^{2}$ are the lowering operators. The other eigenstates are obtained by acting on $|0\rangle$ with rising operators $\hat{s}^{+}(v) \equiv \hat{s}^{1}(v)+i \hat{s}^{2}(v)$,

$$
\left|\tilde{v}_{1}, \ldots, \tilde{v}_{M}\right\rangle=\hat{s}^{+}\left(\tilde{v}_{1}\right) \hat{s}^{+}\left(\tilde{v}_{2}\right) \ldots \hat{s}^{+}\left(\tilde{v}_{M}\right)|0\rangle
$$

at certain points $\tilde{v}_{m}$ which satisfy the Bethe equations,

$$
-\sum_{n=1}^{N} \frac{s_{n}}{\tilde{v}_{m}-v_{n}}+\sum_{\substack{m^{\prime}=1 \\ m^{\prime} \neq m}}^{M} \frac{1}{\tilde{v}_{m}-\tilde{v}_{m^{\prime}}}=0
$$

The eigenvalue of $\hat{\mathcal{T}}(v)$ corresponding to the state (3.19) is

$$
\mathcal{T}(v)=W^{2}+\partial_{v} W, \quad W(v)=-\sum_{n=1}^{N} \frac{s_{n}}{v-v_{n}}+\sum_{m=1}^{M} \frac{1}{v-\tilde{v}_{m}} .
$$

To sum up, one solves eqs. (3.20) for every $M$ and finds all $\prod_{n}\left(2 s_{n}+1\right)$ eigenvectors and eigenvalues of $\hat{\mathcal{T}}(v)$.

Importantly, the Bethe equations (3.20) coincide with the algebraic equations (3.12) for the parameters $\tilde{v}_{m}$ of the solitons in dilaton gravity. This establishes one-to-one correspondence between our exact solutions and the eigenstates (3.19) of the Gaudin model. The singularities $\left\{s_{n}, v_{n}\right\}$ and zeros $\left\{\tilde{v}_{m}\right\}$ of $\psi(v)$ are related to the parameters of the Gaudin Hamiltonians (3.16) and Bethe states (3.19), respectively. Besides, the incoming flux $T_{v v}(v)$ is proportional to the eigenvalue of $\hat{\mathcal{T}}(v): T_{v v}(v)=-2 \mathrm{e}^{-2 \phi_{0}} \mathcal{T}(v)$, cf. eqs. (3.2), (3.11) and (3.21). The related quantities of the two models are listed in table 2.

One can use the Gaudin model to study solitons in dilaton gravity. We are interested in the case of well-localized $T_{v v}=O\left(v^{-4}\right)$ as $v \rightarrow+\infty$. The corresponding Gaudin states have zero total $\operatorname{spin}^{13}$

$$
\hat{\boldsymbol{S}}=\sum_{n} \hat{\boldsymbol{s}}_{n}
$$

\footnotetext{
${ }^{13}$ Note that $\hat{\boldsymbol{S}}$ commutes with all Gaudin Hamiltonians.
} 
because $\hat{\mathcal{T}} \rightarrow(\hat{\boldsymbol{S}} / v)^{2}$ as $v \rightarrow+\infty$, see eq. (3.17). Using this property, one counts the number of solitons with correct asymptotics by adding up spins. For example, there are two such solutions with four $s=1 / 2$ singularities because the Hilbert space of four $s=1 / 2$ spins has two-dimensional zero- $\hat{\boldsymbol{S}}$ subspace: $(1 / 2)^{\otimes 4}=0 \oplus 0 \oplus 1 \oplus 1 \oplus 1 \oplus 2$, where the spin representations are marked with their highest weights.

Besides, now we can explain what happens at $v_{1} \rightarrow v_{2}$ when two singularities of the solitons coalesce. In this limit the spin operator (3.17),

$$
\hat{\boldsymbol{s}}(v) \rightarrow \frac{\hat{\boldsymbol{s}}_{1}+\hat{\boldsymbol{s}}_{2}}{v-v_{2}}+\sum_{n \geq 3} \frac{\hat{\boldsymbol{s}}_{n}}{v-v_{n}} \quad \text { as } \quad v_{1} \rightarrow v_{2},
$$

depends on the sum $\hat{s}_{1}+\hat{s}_{2}$. The corresponding solutions have singularities at $v=v_{2}$ of powers $\left|s_{1}-s_{2}\right|,\left|s_{1}-s_{2}\right|+1, \ldots,\left(s_{1}+s_{2}\right)$ in accordance with the irreducible representations of $\hat{\boldsymbol{s}}_{1}+\hat{\boldsymbol{s}}_{2}$. For instance, consider coalescence of two $s_{1,2}=1 / 2$ singularities as $v_{1} \rightarrow v_{2}$. The second-order equations (3.6) at these singularities have four solutions corresponding to four eigenstates of two $s=1 / 2$ spins. In the limit $v_{1} \rightarrow v_{2}$ the spins sum up and we obtain $^{14}$ one $s=0$ (non-singular) solution and three solutions with $s=1$ singularity.

Finally, one can obtain more general solutions with infinite number of singularities using the thermodynamic Bethe Ansatz for the Gaudin model [55].

Example. Consider the solution with four $s=1 / 2$ singularities arranged in two complex conjugate pairs $v_{1,2}=a_{1} \pm i b_{1}, v_{3,4}=a_{2} \pm i b_{2}$. Solving eqs. (3.8), (3.9), (3.6), we obtain, as expected above, two solutions

$$
\begin{aligned}
T_{v v}^{( \pm)}= & \frac{6 b_{1}^{2} \mathrm{e}^{-2 \phi_{0}}}{\left(\left(v-a_{1}\right)^{2}+b_{1}^{2}\right)^{2}}+\frac{6 b_{2}^{2} \mathrm{e}^{-2 \phi_{0}}}{\left(\left(v-a_{2}\right)^{2}+b_{2}^{2}\right)^{2}} \\
& -2 \mathrm{e}^{-2 \phi_{0}} \frac{\left(a_{1}-a_{2}\right)^{2}+b_{1}^{2}+b_{2}^{2} \pm \sqrt{\Delta}}{\left(\left(v-a_{1}\right)^{2}+b_{1}^{2}\right)\left(\left(v-a_{2}\right)^{2}+b_{2}^{2}\right)},
\end{aligned}
$$

where $\Delta=\left(\left(a_{1}-a_{2}\right)^{2}+b_{1}^{2}+b_{2}^{2}\right)^{2}+12 b_{1}^{2} b_{2}^{2}>0$. In the limit $a_{1} \rightarrow a_{2}, b_{1} \rightarrow b_{2}$ the pairs of singularities in the upper and lower parts of the complex $v$-plane coalesce, and one obtains a nonsingular solution and a solution (3.15) with two $s=1$ singularities,

$$
T_{v v}^{(+)} \rightarrow 0, \quad T_{v v}^{(-)} \rightarrow \frac{16 \mathrm{e}^{-2 \phi_{0}} b_{2}^{2}}{\left(\left(v-a_{2}\right)^{2}+b_{2}^{2}\right)^{2}},
$$

again in accordance with the above expectations.

Note that $T_{v v}^{(+)}(v)$ is not positive-definite at real positive $v$ and therefore unphysical. The function $T_{v v}^{(-)}(v)$ describes incoming matter flux with two peaks at $v \sim a_{1}$ and $a_{2}$, see figure 7 .

\subsection{Positivity condition}

Physical solutions have real $\psi(v)$ at real $v$. Thus, their singularities $v_{n}$ and zeros $\tilde{v}_{m}$ are either real or organized in complex conjugate pairs like in figure 6 . Besides, the singularities $v_{n}$ may not be placed at the physical part $v \geq 0$ of the real axis.

\footnotetext{
${ }^{14}$ One can explicitly demonstrate this by solving eqs. (3.6) to the leading order in $v_{1}-v_{2} \rightarrow 0$.
} 


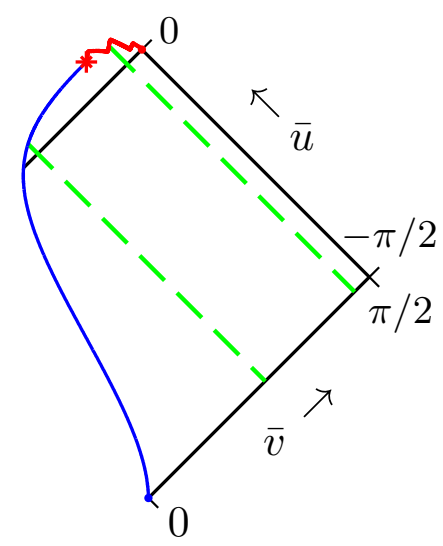

Figure 7. Solution "_" in eq. (3.22) with four $s=1 / 2$ poles and parameters $\lambda a_{1}=-0.1, \lambda b_{1}=1$, $\lambda a_{2}=0.2, \lambda b_{2}=10$, and $\mathrm{e}^{-2 \phi_{0}}=1$. The two peaks of the incoming matter flux are marked by the dashed lines. For this choice of parameters, the second peak forms the black hole.

The remaining nontrivial condition is $T_{v v}(v) \geq 0$ at $v \geq 0$, eq. (3.3). This inequality is not satisfied automatically. For example, our solutions with two singularities (3.15) have negative and positive $T_{v v}(v)$ at $v_{1,2}<0$ and $v_{1,2}=a \pm i b$, respectively. In fact, any solution with all singularities placed at $v<0$ is unphysical. In this case the operator $\hat{\boldsymbol{s}}(v)$ at real $v$ is Hermitean, and therefore $\hat{\mathcal{T}}(v)$ in eq. (3.17) has positive-definite eigenvalues $\mathcal{T}(v) \propto-T_{v v}(v)$.

In the opposite case when all singularities are organized in complex conjugate pairs $v_{2 k-1}, v_{2 k}=a_{k} \pm i b_{k}$ with $s_{2 k-1}=s_{2 k}$, one expects to find at least one physical solution. Indeed, consider the state $\left|\Psi_{1}\right\rangle$ (not an eigenstate) of the Gaudin model satisfying $\left(\hat{s}_{2 k-1}+\hat{s}_{2 k}\right)\left|\Psi_{1}\right\rangle=0$ for all $k$. Explicit calculation shows that $\left\langle\Psi_{1}|\hat{\mathcal{T}}(v)| \Psi_{1}\right\rangle<0$ at real $v$. On the other hand, the variational principle implies that for any $N$ real points $w_{n}$ there exists an eigenstate $|\Psi\rangle$ minimizing all $\left\langle\Psi\left|\hat{\mathcal{T}}\left(w_{n}\right)\right| \Psi\right\rangle$. The respective eigenvalue $\mathcal{T}(v)$ is negative at all $v=w_{n}$ suggesting that $T_{v v}(v) \propto-\mathcal{T}(v)$ is positive at the entire real axis.

Let us explicitly select the above physical solution at $b_{k} \rightarrow 0$. In this case $T_{v v}(v)$ falls into a collection of peaks at $v \sim a_{k}$ near the singularities $v_{2 k-1}, v_{2 k}$. At $\left|v-a_{k}\right| \gg b_{k}$ and yet, far away from other singularities, the operator (3.17) takes the form $\hat{\mathcal{T}}(v) \approx$ $\left(\hat{\boldsymbol{s}}_{2 k-1}+\hat{\boldsymbol{s}}_{2 k}\right)^{2} /\left(v-a_{k}\right)^{2}$. Its eigenvalue $\mathcal{T}(v) \propto-T_{v v}(v)$ is positive-definite unless the eigenstate satisfies $\left(\hat{\boldsymbol{s}}_{2 k-1}+\hat{\boldsymbol{s}}_{2 k}\right)|\Psi\rangle=0$. Thus, in the limit $b_{k} \rightarrow 0$ the physical eigenstate coincides with the state $\left|\Psi_{1}\right\rangle$ introduced above. The respective energy flux $T_{v v}(v)$ is the sum of two-spin terms (3.15),

$$
T_{v v} \approx 8 \mathrm{e}^{-2 \phi_{0}} \sum_{k=1}^{N / 2} \frac{s_{2 k}\left(s_{2 k}+1\right) b_{k}^{2}}{\left[\left(v-a_{k}\right)^{2}+b_{k}^{2}\right]^{2}} \quad \text { at small } b_{k} .
$$

One expects that this solution remains physical at finite $b_{k}$. 


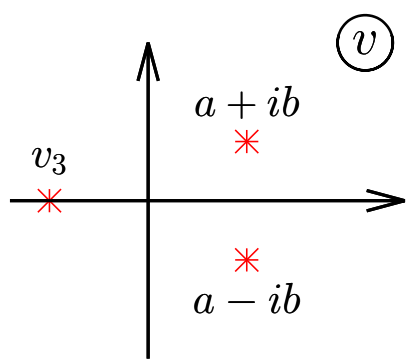

(a)

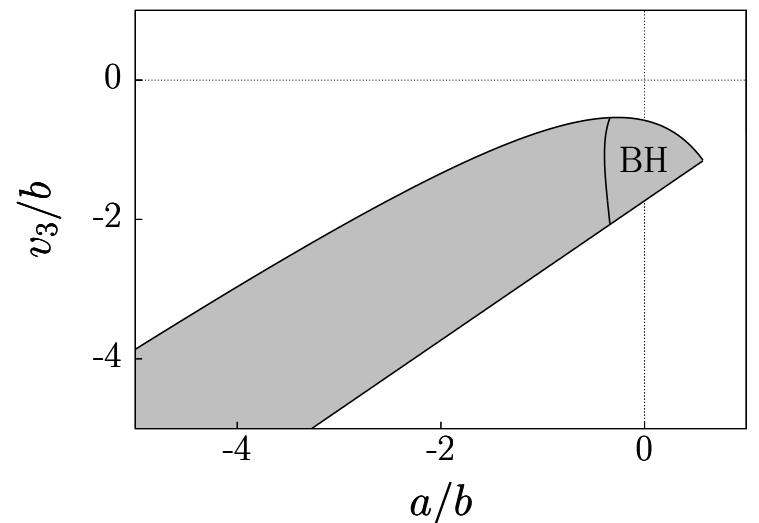

(b)

Figure 8. (a) Singularities of the solution (3.24). (b) Parameters of this solution giving positivedefinite $T_{v v}(v)$ at $v \geq 0$ (gray region). The upper right corner of this region corresponds to black hole formation.

Example. In general case the positivity condition bounds parameters of the solutions. Consider e.g. the soliton with three $s=1$ singularities at $v_{1,2}=a \pm i b, v_{3}<0$, see figure 8a. Solving eqs. (3.8), (3.9), one obtains,

$$
T_{v v}=\frac{16 \mathrm{e}^{-2 \phi_{0}} b^{2}}{\left((v-a)^{2}+b^{2}\right)^{2}}-\frac{4 \mathrm{e}^{-2 \phi_{0}}\left[\left(a-v_{3}\right)^{2}+b^{2}\right]}{\left(v-v_{3}\right)^{2}\left[(v-a)^{2}+b^{2}\right]} .
$$

The second (negative) term in this expression represents contribution of the singularity $v_{3}<0$. It can be compensated by the first term if the singularities $v_{1}$ and $v_{2}$ are close enough to $v_{3}$. Namely, the function (3.24) is positive-definite at $v \geq 0$ if $a-b \sqrt{3} \leq v_{3} \leq$ $\left(a^{2}+b^{2}\right) /(a-b \sqrt{3})$, see the gray region in figure $8 \mathrm{~b}$. The solutions with these parameters involve one peak of the incoming flux, just like the solutions (3.15).

\section{Critical chaos}

\subsection{Perturbative expansion in the critical regime}

In section 2.3 we argued that the critical solutions at the verge of black hole formation have constant $\psi(v)$ and null boundary $U(v)$ at large $v$, see figure 4 . One can say that they describe formation of the minimal-mass black holes with the boundary placed precisely at the horizon [46, 56], cf. [57, 58].

At energies somewhat below critical the boundary has long almost null part ("plateau"), see figure 9a. The energy flux reflected from this part is strongly amplified by the Lorentz factor of the boundary and forms a high and narrow peak in $T_{u u}(u)$, see figure $9 \mathrm{~b}$. We will argue below that in the critical limit the peak tends to a $\delta$-function (shock-wave) with energy equal to the minimal black hole mass $M_{c r}$. In the overcritical solutions the shock-wave is swallowed by the black hole. Besides, we will see in the next 

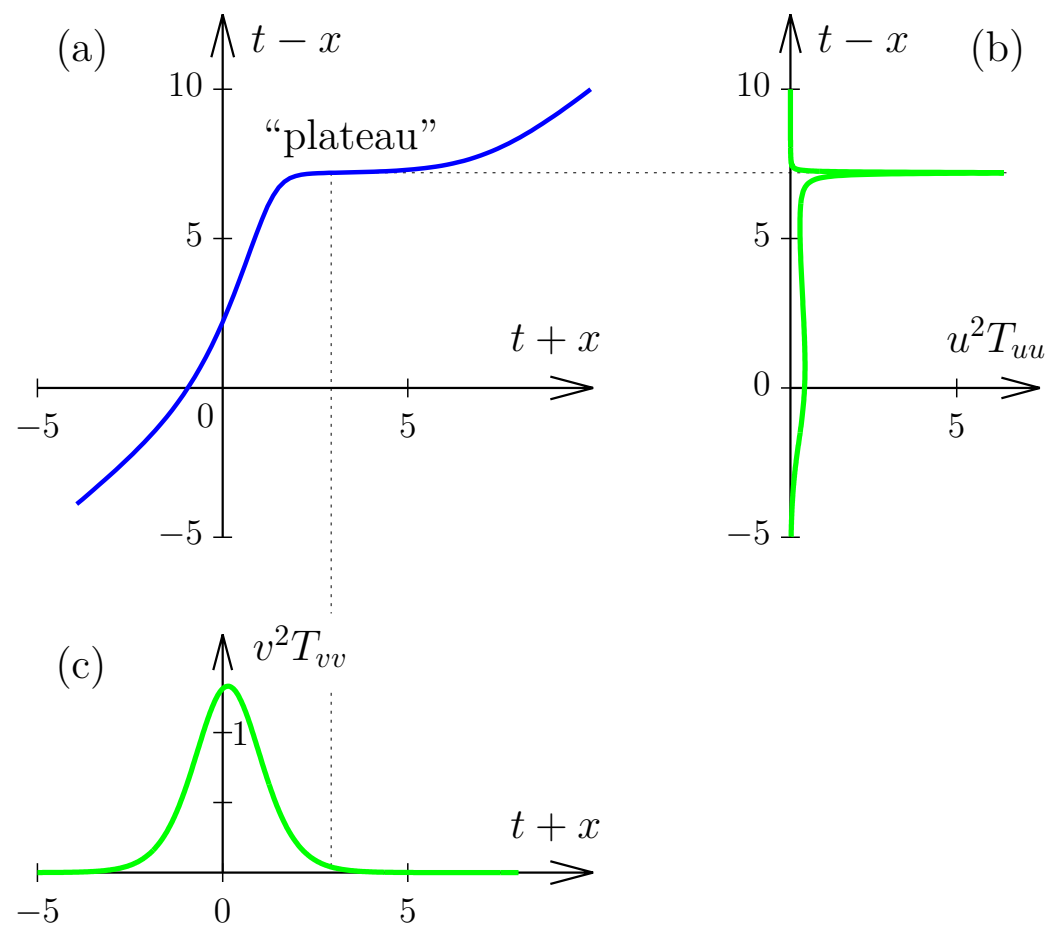

Figure 9. Solution (3.15) at almost critical values of parameters $s=b=\mathrm{e}^{-2 \phi_{0}}=1$ and $a=$ $a_{c r}-10^{-3}$, where $a_{c r}=-1 / \sqrt{3}$ and we use units with $\lambda=1$. In this case $C \approx 7 \times 10^{-4} \ll 1$. Figure (a) shows the boundary $u=U(v)$ in the asymptotically flat light-cone coordinates $t+x=\log (\lambda v) / \lambda$, $t-x=-\log (-\lambda u) / \lambda$, see eq. (2.11). In figures (b), (c) we plot the outgoing and incoming energy fluxes $u^{2} T_{u u}$ and $v^{2} T_{v v}$ as functions of $t-x$ and $t+x$, respectively.

section that the structure of the peak is highly sensitive to the initial data. This feature impedes global integrability of the model.

Let us find the boundary $U(v)$ in the "plateau" region where $v$ is large and $T_{v v}(v)$ is small. In this case eq. (2.13) can be solved perturbatively by representing $\psi=1+\psi^{(1)}+$ $\psi^{(2)}+\ldots$, where $\psi^{(k)} \propto\left(T_{v v}\right)^{k}$. Using $\psi \approx 1$ in the r.h.s. of eq. (2.13), we obtain,

$$
\psi^{(1)}(v)=C v+e^{2 \phi_{0}}\left[g(v)-g_{\infty}\right],
$$

where the function $g(v)$ is introduced in eq. (2.8) and $g_{\infty}$ is its value at $v \rightarrow+\infty$. Note that the linear asymptotics $C v \ll 1$ of the solution appears at first order of expansion in eq. (4.1) because in the near-critical regime $\partial_{v} \psi \approx C$ is small at large $v$. In what follows we will regard $C$ as a parameter of the expansion. Using $\psi \approx 1+\psi^{(1)}$ in the r.h.s. of eq. (2.13), we get

$$
\partial_{v} \psi^{(2)}(v)=\mathrm{e}^{2 \phi_{0}}\left(g-g_{\infty}\right)\left(\mathrm{e}^{2 \phi_{0}} \partial_{v} g-C\right)+\mathrm{e}^{2 \phi_{0}} C v \partial_{v} g+e^{4 \phi_{0}} \int_{v}^{\infty} d v^{\prime}\left(\partial_{v^{\prime}} g\right)^{2}
$$

The higher-order corrections $\psi^{(n)}$ are obtained in similar way. 
Now, we compute the reflected energy flux $T_{u u}(u)$ and the boundary function $U(v)$ using eqs. (2.14) and (2.12),

$$
\begin{aligned}
T_{u u}(U(v)) & \approx \frac{\lambda^{4} \mathrm{e}^{4 \phi_{0}} T_{v v}(v)}{\left[C+e^{2 \phi_{0}} \partial_{v} g(v)\right]^{4}}, \\
\lambda^{2} U(v) & \approx-\mathrm{e}^{-2 \phi_{0}} C+\mathrm{e}^{-2 \phi_{0}} C^{2} v+2 C\left(g-g_{\infty}\right)-\mathrm{e}^{2 \phi_{0}} \int_{v}^{\infty} d v^{\prime}\left(\partial_{v^{\prime}} g\right)^{2} .
\end{aligned}
$$

We kept one and two orders of the expansion in eqs. (4.2) and (4.3), respectively. Note that the leading (first) term in $U(v)$ is constant; this behavior corresponds to the "plateau" in figure 9a. At the same time, the reflected flux (4.2) has a peak at large $v$ corresponding to $\partial_{v} g \sim C \mathrm{e}^{-2 \phi_{0}}$. This peak is narrow in terms of slowly-changing $u=U(v)$ in agreement with figure $9 \mathrm{~b}$.

Using the soliton asymptotics $T_{v v} \propto v^{-4}$ and $\partial_{v} g \propto v^{-3}$, one finds that the peak in eq. (4.2) occurs at $v \propto C^{-1 / 3}$, and its width $\Delta v$ is of the same order. The respective value of $U(v)$ is approximately given by the first term in eq. (4.3), while the peak width $\Delta U \propto C^{2 / 3} U$ is controlled by the second-order terms. In the critical limit $C \rightarrow 0$ the peak of $T_{u u}(u)$ is infinitely high and narrow.

Calculating the total energy within the shock-wave at $C \rightarrow 0$, we obtain,

$$
E_{\text {peak }}=\lambda \int_{u \sim C}|u| d u T_{u u}(u) \rightarrow-2 \lambda C \int_{0}^{+\infty} \frac{d v \partial_{v}^{2} g(v)}{\left[C+e^{2 \phi_{0}} \partial_{v} g(v)\right]^{2}} \rightarrow 2 \lambda \mathrm{e}^{-2 \phi_{0}}
$$

where eqs. (4.2), (4.3) were used. The value of $E_{\text {peak }}$ coincides with the minimal black hole mass $M_{c r}$ implying that the peak of $T_{u u}(u)$ tends to a $\delta$-function in the critical limit.

\subsection{Shock-wave instability}

Since our model is equipped with the general solution, one may think that it is integrable, i.e. has a complete set of conserved quantities $\left\{I_{k}\right\}$ smoothly foliating the phase space. In the in-sector these quantities are arbitrary functionals $I_{k}\left[f_{\text {in }}\right]$ of conserved $f_{\text {in }}(v)$, cf. [59]. Then, $I_{k}$ can be computed at arbitrary space-like line: to this end one evolves the classical fields from this line to $J^{-}$, extracts the incoming wave ${ }^{15} f_{\text {in }}(v)$, and calculates $I_{k}\left[f_{\text {in }}\right]$. The quantities $\left\{I_{k}\right\}$ obtained in this way are conserved by definition. For example, in the out-sector one gets $I_{k}\left[f_{\text {out }}\right] \equiv I_{k}\left[f_{\text {in }}\right]$ if $f_{\text {out }}(u)$ and $f_{\text {in }}(v)$ are related by classical evolution, eq. (2.9).

Let us argue, however, that $\left\{I_{k}\right\}$ cannot be smoothly defined in the near-critical regime because the map $f_{\text {in }} \rightarrow f_{\text {out }}$ in this case is essentially singular. To simplify the argument, we consider solutions with the modulated flux at large $v$,

$$
T_{v v}=\left(\partial_{v} f_{\text {in }}\right)^{2}, \quad \partial_{v} f_{\text {in }} \approx A v^{-2} \cos (\omega \ln (\lambda v)) \quad \text { at } \quad v \gtrsim C^{-1 / 3},
$$

where $C$ is the small parameter of the near-critical expansion. If $\omega$ is small as well, the asymptotics of $T_{v v}$ is almost power-law, like in the ordinary solitons. However, the shockwave part of the reflected flux represents squeezed and amplified tail of $T_{v v}$ at $v \sim C^{-1 / 3}$,

\footnotetext{
${ }^{15}$ Recall that all our solutions start from flat space-time in the infinite past.
} 


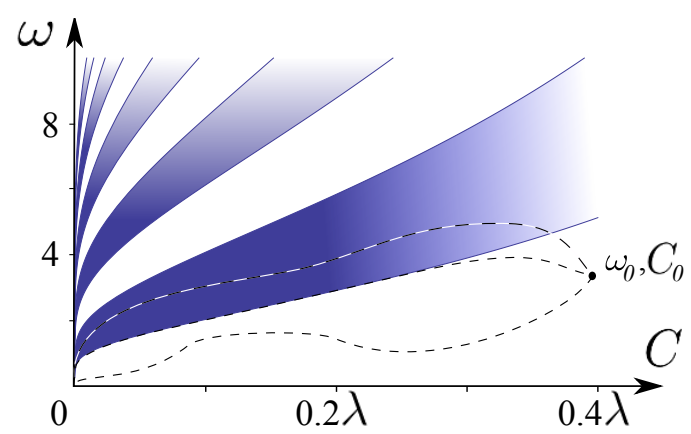

Figure 10. Regions $\Delta \mathcal{I}_{3}>0$ (white) and $\Delta \mathcal{I}_{3}<0$ (blue) in the $(C, \omega)$ plane. We use the solution (4.4) with $A^{2}=12 \mathrm{e}^{-2 \phi_{0}} / \lambda^{2}$.

see figure 9. It should be essentially modulated. For simplicity, let us characterize the outgoing wave packet with a single quantity

$$
\begin{aligned}
\mathcal{I}_{3}(C, \omega) & \equiv \int_{-\infty}^{+\infty} d(t-x)\left(\partial_{t-x} f_{\text {out }}\right)^{3}=\Delta \mathcal{I}_{3}(C, \omega)+\text { const }, \\
\Delta \mathcal{I}_{3} & =\int_{0}^{\infty} d v \frac{C^{2}\left(\partial_{v} f_{\text {in }}\right)^{3}}{\left[C+e^{2 \phi_{0}} g_{v}(v)\right]^{4}},
\end{aligned}
$$

where we used the flat coordinates (2.11) in the definition of $\mathcal{I}_{3}$, then separated the shock-wave part $\Delta \mathcal{I}_{3}$ of the integral at $t-x \equiv-\log (-\lambda u) / \lambda \gtrsim \log C$ from the $(C, \omega)$ independent contribution at smaller $t-x$. In the second line we substituted the shockwave profile (4.2), (4.3) and extended the integration range to $v \geq 0$. Now, one substitutes the asymptotics (4.4) into eq. (4.5) and finds that $\Delta \mathcal{I}_{3}(C, \omega)$ is quasi-periodic. Indeed, change of the integration variable $v \mapsto v e^{2 \pi n / \omega}$ with integer $n$ gives relation ${ }^{16}$ $\Delta \mathcal{I}_{3}\left(e^{6 \pi n / \omega} C, \omega\right)=e^{-2 \pi n / \omega} \Delta \mathcal{I}_{3}(C, \omega)$. Thus, $\Delta \mathcal{I}_{3}=C^{-1 / 3} \mathcal{J}(\omega \log C)$, where $\mathcal{J}(x)$ is $6 \pi$-periodic.

We see that $\Delta \mathcal{I}_{3}$ has an essential singularity at $\omega=C=0$. Indeed, taking the limit $C \rightarrow 0$ along the paths $\omega \log C=$ const, one obtains $\Delta \mathcal{I}_{3} \rightarrow-\infty, 0$, or $+\infty$, see figure 10 . Thus, any value of $\Delta \mathcal{I}_{3}$ can be obtained by adjusting the limiting path.

The above property ascertains dynamical chaos in the critical limit of our model. Indeed, infinitesimally small changes (4.4) of the initial data at small $C$ produce outgoing fluxes with essentially different values of $\mathcal{I}_{3}$. This prevents one from characterizing the critical evolution with a set of smooth conserved quantities $I_{k}$. Indeed, all functionals $I_{k}\left[f_{\text {in }}\right]$, being smooth in the in-sector, are not sensitive to $\omega$ at small values of latter. Thus, they fail to describe essentially different out-states $f_{\text {out }}(u)$ at different $\omega$. From a more general perspective, one can introduce the integrals which are smooth either in the in-sector or in the out-sector, but not in both.

\footnotetext{
${ }^{16}$ In this case $g^{\prime}\left(v \mathrm{e}^{2 \pi n / \omega}\right)=\mathrm{e}^{-6 \pi n / \omega} g^{\prime}(v)$, where the derivative is taken with respect to the argument, see eqs. (2.8).
} 


\section{Discussion}

In this paper we considered two-dimensional CGHS model with a regulating dynamical boundary [33, 34]. This model is weakly coupled and causally similar to the sphericallysymmetric gravity in many dimensions. We demonstrated that classical field equations in this model are exactly solvable. We constructed their general solution and studied in detail a large subset of soliton solutions with transparent properties. We illustrated the results with many explicit examples hoping that this model will serve as a practical playground for black hole physics.

In the critical regime i.e. at the verge of black hole formation, our model displays dynamical instabilities specific to chaotic systems. This property is similar to the nearhorizon chaos suggested recently in the context of AdS/CFT correspondence [23-28]. We argued that it hinders global integrability of the model.

We see several applications of our results. First, exact solvability may extend to oneloop semiclassical level if one adds a reflective boundary to the RST model [5]. This approach, if successful, will produce analytic solutions describing black hole formation and evaporation. The singularities of such solutions should be either covered by the boundary or hidden behind the space-like line $\phi=\phi_{0}$, see figure $4 \mathrm{~b}$. Then a complete Penrose diagram for the evaporation process may be obtained, cf. [39, 41, 42, 46].

Second, in the alternative approach one directly adds one-loop corrections to the classical equations of our model with a boundary and integrates the resulting system numerically, cf. $[60,61]$. By the same reasons as above, the respective solutions should completely describe the process of black hole evaporation.

Third and finally, the model of this paper is ideal for applying the semiclassical method of $[62,63]$ which relates calculation of the exponentially suppressed $S$-matrix elements to certain complex classical solutions. The results of such calculations may be used to test unitarity of the gravitational $S$-matrix [63].

\section{Acknowledgments}

We thank S. Sibiryakov for participating at early stages of this project. We are grateful to École Polytechnique Fédérale de Lausanne for hospitality during our visits. This work was supported by the grant RSCF 14-22-00161.

\section{A Field equations and boundary conditions}

\section{A.1 Derivation}

Field equations in the bulk are obtained by varying the action (2.1) with respect to $g_{\mu \nu}$, $\phi$, and $f$, and ignoring the boundary terms,

$$
\begin{aligned}
4 e^{-2 \phi} \nabla_{\mu} \nabla_{\nu} \phi+4 g_{\mu \nu} e^{-2 \phi}\left[(\nabla \phi)^{2}-\nabla^{2} \phi-\lambda^{2}\right] & =\nabla_{\mu} f \nabla_{\nu} f-\frac{1}{2} g_{\mu \nu}(\nabla f)^{2}, \\
(\nabla \phi)^{2}-\nabla^{2} \phi-\lambda^{2} & =R / 4 \\
\nabla^{2} f & =0
\end{aligned}
$$


The first line here relates the energy-momentum tensors of $\phi$ and $f,-T_{\mu \nu}^{(\phi)}=T_{\mu \nu}^{(f)}$. The second line implies, in addition, that the rescaled metric $\mathrm{e}^{-2 \phi} g_{\mu \nu}$ is flat.

To find the boundary conditions at the line $\phi=\phi_{0}$, we keep the boundary terms in the variation of the action. For a start, let us consider variations preserving the coordinate position of the boundary $\phi=\phi_{0}$. We take $\delta \phi=0$ along this line and fix the direction of its outer normal, $\delta n_{\mu} \propto n_{\mu}$. The integration domains in eq. (2.1) are unchanged by such variations. One obtains,

$$
\delta S=-\int_{\phi=\phi_{0}} d \tau\left[2 h^{\mu \nu} \delta h_{\mu \nu} \mathrm{e}^{-2 \phi_{0}}\left(n^{\kappa} \nabla_{\kappa} \phi-\lambda\right)+\delta f n^{\kappa} \nabla_{\kappa} f\right]=0,
$$

where we canceled the bulk terms using eqs. (A.1)-(A.3) and introduced the induced metric $h_{\mu \nu} \equiv g_{\mu \nu}-n_{\mu} n_{\nu}$. The variation (A.4) gives the boundary conditions (2.2). Note that the space-time is flat near the boundary: one obtains $R=0$ at $\phi=\phi_{0}$ using the first of eqs. (2.2), eq. (A.2) and the trace of eq. (A.1).

Now, let us consider general variations shifting the position of the boundary. They are combinations of the general coordinate transformations and position-preserving variations considered above. The action is unchanged by these variations: it is covariant and already extremized at fixed coordinate position of the boundary.

\section{A.2 Solution in the conformal gauge}

Let us review the general solution [4] of the bulk equations (A.1)-(A.3), see [1, 2] for details.

In the light-cone frame (2.4) eq. (A.3) takes the form $\partial_{u} \partial_{v} f=0$, its solution is given by eq. (2.5). Combining eq. (A.2) with the trace of eq. (A.1) and substituting $R=8 \mathrm{e}^{-2 \rho} \partial_{u} \partial_{v} \rho$, we obtain,

$$
\partial_{u} \partial_{v}(\phi-\rho)=0 \quad \Rightarrow \quad \phi=\rho,
$$

where the residual coordinate freedom ${ }^{17}$ was fixed in the last equation. After that eqs. (A.1), namely,

$$
\begin{aligned}
\partial_{u}^{2} \mathrm{e}^{-2 \phi} & =-\frac{1}{2}\left(\partial_{u} f\right)^{2}, \\
\partial_{v}^{2} \mathrm{e}^{-2 \phi} & =-\frac{1}{2}\left(\partial_{v} f\right)^{2}, \\
\partial_{u} \partial_{v} \mathrm{e}^{-2 \phi} & =-\lambda^{2},
\end{aligned}
$$

are integrated into

$$
\mathrm{e}^{-2 \rho}=\mathrm{e}^{-2 \phi}=\frac{M_{-}}{2 \lambda}-\lambda^{2}\left(u-u_{0}\right)\left(v-v_{0}\right)+g(v)+h(u) .
$$

In this expression $M_{-}, u_{0}$, and $v_{0}$ are integration constants; functions $g(v)$ and $h(u)$ were introduced in eq. (2.8). We fix $u_{0}=v_{0}=0$ by shifting $u$ and $v$. After that $M_{-}$represents the mass of white hole in the infinite past $[1,2]$. Indeed, the past time infinity $i^{-}$in

\footnotetext{
${ }^{17}$ Namely, the transformations $u \rightarrow \tilde{u}(u), v \rightarrow \tilde{v}(v)$ preserving the metric (2.4).
} 
figure $1 \mathrm{~b}$ is reached at $u \rightarrow-\infty, v \rightarrow 0$, and constant $\phi$, cf. eq. (2.3). If $M_{-} \neq 0$, the curvature remains nonzero in this limit,

$$
R=4 \mathrm{e}^{2 \rho}\left(\partial_{u} \mathrm{e}^{-2 \rho}\right)\left(\partial_{v} \mathrm{e}^{-2 \rho}\right)-4 \partial_{u} \partial_{v} \mathrm{e}^{-2 \rho} \rightarrow 2 \lambda \mathrm{e}^{2 \phi} M_{-},
$$

where eq. (A.5) with $u_{0}=v_{0}=0$ was used. In this paper we consider solutions starting from flat space-time. Thus, $M_{-}=0$, and eq. (A.5) takes the form (2.7).

It is worth noting that the patch $u \in(-\infty, 0)$ and $v \in(0,+\infty)$ covers all space-time accessible to the outside observer. Indeed, we already mentioned that the time infinities $i^{-}$ and $i^{+}$are reached in the limits $u \rightarrow-\infty$ and $v \rightarrow+\infty$ at finite values of the dilaton field $\phi$. By eq. (2.7), the product $u v$ remains finite in these limits implying $v \rightarrow+0$ as $u \rightarrow-\infty$ $\left(i^{-}\right)$and $u \rightarrow-0$ as $v \rightarrow+\infty\left(i^{+}\right)$, see figure 3 .

We proceed by deriving equation of motion for the boundary $u=U(v)$ satisfying $\phi(U(v), v)=\phi_{0}$. Taking the derivative of eq. (2.7) along this line, we find,

$$
0=\frac{d}{d v} \mathrm{e}^{-2 \phi_{0}}=U^{\prime}\left[\partial_{u} h-\lambda^{2} v\right]+\partial_{v} g-\lambda^{2} U, \quad \text { at } \quad u=U(v),
$$

where $U^{\prime} \equiv d U / d v>0$ because the boundary is time-like. The other two equations come from the boundary conditions (2.2). Introducing the unit outer normal

$$
n^{u}=\mathrm{e}^{-\phi_{0}} \sqrt{U^{\prime}}, \quad n^{v}=-\mathrm{e}^{-\phi_{0}} / \sqrt{U^{\prime}}
$$

and using eq. (A.6), we rewrite eqs. (2.2) in the form (2.9).

At this point, we have three equations, eqs. (A.6) and (2.9), for the two unknown functions $f_{\text {out }}(u)$ and $U(v)$. Note, however, that eq. (A.6) follows from the other two equations. Indeed,

$$
\frac{d}{d v}\left(\frac{\partial_{v} g-\lambda^{2} U}{U^{\prime}}\right)=\lambda^{2} \mathrm{e}^{-2 \varphi_{0}} \frac{d}{d v}\left(\partial_{v} g-\lambda^{2} U\right)^{-1}=\frac{\left(\partial_{v} f_{\text {in }}\right)^{2} / 2+\lambda^{2} U^{\prime}}{U^{\prime}}=\frac{d}{d v}\left(\lambda^{2} v-\partial_{u} h\right),
$$

where we expressed $U^{\prime}$ and $g$ via eqs. (2.9) and (2.8) in the first and second equalities, then turned $f_{\text {in }} \rightarrow f_{\text {out }}$ by the second of eqs. (2.9) and used the equation for $U^{\prime}$, again. One concludes that eq. (A.6) is automatically satisfied once the initial conditions for $U(v)$ are chosen correctly.

\section{B Bethe Ansatz for the Gaudin model}

In this appendix we review Bethe Ansatz for the Gaudin model (3.16), see [47-49] for details.

One introduces raising and lowering operators $\hat{s}^{ \pm}(v)=\hat{s}^{1}(v) \pm i \hat{s}^{2}(v)$ for the positiondependent spin (3.17). The commutation rules of these operators are

$$
\left[\hat{s}^{-}(v), \hat{s}^{+}(w)\right]=2 \frac{\hat{s}^{3}(v)-\hat{s}^{3}(w)}{v-w}, \quad\left[\hat{s}^{3}(v), \hat{s}^{ \pm}(w)\right]=\mp \frac{\hat{s}^{ \pm}(v)-\hat{s}^{ \pm}(w)}{v-w} .
$$

The Hamiltonian $\hat{\mathcal{T}}(v)$ in eq. (3.17) takes the form

$$
\hat{\mathcal{T}}(v)=\frac{1}{2} \hat{s}^{+}(v) \hat{s}^{-}(v)+\frac{1}{2} \hat{s}^{-}(v) \hat{s}^{+}(v)+\left(\hat{s}^{3}(v)\right)^{2} .
$$


Now, it is straightforward to check that the spin-down state (3.18) is an eigenstate:

$$
\hat{\mathcal{T}}(v)|0\rangle=\left[\left(W_{0}\right)^{2}+\partial_{v} W_{0}\right]|0\rangle, \quad \text { where } \quad W_{0}(v)=-\sum_{n} \frac{s_{n}}{v-v_{n}}
$$

is the eigenvalue of the third spin component, $\hat{s}^{3}(v)|0\rangle=W_{0}(v)|0\rangle$.

One explicitly acts with $\hat{\mathcal{T}}(v)$, eq. (B.1), on the state (3.19) and obtains,

$$
\hat{\mathcal{T}}(v)\left|\tilde{v}_{1}, \ldots, \tilde{v}_{M}\right\rangle=\mathcal{T}(v)\left|\tilde{v}_{1}, \ldots, \tilde{v}_{M}\right\rangle-\sum_{m=1}^{M} \frac{2 L_{m}}{v-\tilde{v}_{m}}\left|\tilde{v}_{1}, \ldots, \tilde{v}_{m} \mapsto v, \ldots, \tilde{v}_{M}\right\rangle,
$$

where $\mathcal{T}(v)$ is given by eq. (3.21), $L_{m}$ is the left-hand side of eq. (3.20), and arrow denotes substitution. Note that the relations

$$
\begin{aligned}
{\left[\hat{\mathcal{T}}(v), \hat{s}^{+}(w)\right] } & =\frac{2}{v-w}\left(\hat{s}^{+}(w) \hat{s}^{3}(v)-\hat{s}^{+}(v) \hat{s}^{3}(w)\right), \\
\hat{s}^{3}(v)\left|\tilde{v}_{1}, \ldots, \tilde{v}_{M}\right\rangle & =W(v)\left|\tilde{v}_{1}, \ldots, \tilde{v}_{M}\right\rangle-\sum_{m} \frac{1}{v-\tilde{v}_{m}}\left|\tilde{v}_{1}, \ldots, \tilde{v}_{m} \rightarrow v, \ldots, \tilde{v}_{M}\right\rangle,
\end{aligned}
$$

where $W(v)$ is defined in eq. (3.21), are helpful for deriving eq. (B.2).

We conclude that eq. (B.2) coincides with the eigenproblem for $\hat{\mathcal{T}}(v)$ if the Bethe equations $L_{m}=0$, eqs. (3.20), are satisfied. In this case the Bethe states (3.19) are the eigenstates of the Gaudin Hamiltonians (3.16). Moreover, one can prove [47-49] that the basis (3.19) is complete.

Open Access. This article is distributed under the terms of the Creative Commons Attribution License (CC-BY 4.0), which permits any use, distribution and reproduction in any medium, provided the original author(s) and source are credited.

\section{References}

[1] S.B. Giddings, Quantum mechanics of black holes, hep-th/9412138 [INSPIRE].

[2] A. Strominger, Les Houches lectures on black holes, hep-th/9501071 [INSPIRE].

[3] D. Grumiller, W. Kummer and D.V. Vassilevich, Dilaton gravity in two-dimensions, Phys. Rept. 369 (2002) 327 [hep-th/0204253] [INSPIRE].

[4] C.G. Callan Jr., S.B. Giddings, J.A. Harvey and A. Strominger, Evanescent black holes, Phys. Rev. D 45 (1992) R1005 [hep-th/9111056] [InSPIRE].

[5] J.G. Russo, L. Susskind and L. Thorlacius, The Endpoint of Hawking radiation, Phys. Rev. D 46 (1992) 3444 [hep-th/9206070] [INSPIRE].

[6] S.B. Giddings, The gravitational S-matrix: Erice lectures, Subnucl. Ser. 48 (2013) 93 [arXiv: 1105.2036] [INSPIRE].

[7] G. 't Hooft, The Scattering matrix approach for the quantum black hole: An Overview, Int. J. Mod. Phys. A 11 (1996) 4623 [gr-qc/9607022] [INSPIRE].

[8] G. Dvali, C. Gomez, R.S. Isermann, D. Lüst and S. Stieberger, Black hole formation and classicalization in ultra-Planckian $2 \rightarrow N$ scattering, Nucl. Phys. B 893 (2015) 187 [arXiv: 1409.7405] [INSPIRE]. 
[9] S.W. Hawking, Particle Creation by Black Holes, Commun. Math. Phys. 43 (1975) 199 [Erratum ibid. 46 (1976) 206] [INSPIRE].

[10] S.W. Hawking, Breakdown of Predictability in Gravitational Collapse, Phys. Rev. D 14 (1976) 2460 [INSPIRE].

[11] J.M. Maldacena, The Large- $N$ limit of superconformal field theories and supergravity, Int. J. Theor. Phys. 38 (1999) 1113 [hep-th/9711200] [INSPIRE].

[12] J.M. Maldacena, Eternal black holes in anti-de Sitter, JHEP 04 (2003) 021 [hep-th/0106112] [INSPIRE].

[13] A. Almheiri, D. Marolf, J. Polchinski and J. Sully, Black Holes: Complementarity or Firewalls?, JHEP 02 (2013) 062 [arXiv: 1207.3123] [INSPIRE].

[14] A. Almheiri, D. Marolf, J. Polchinski, D. Stanford and J. Sully, An Apologia for Firewalls, JHEP 09 (2013) 018 [arXiv: 1304.6483] [INSPIRE].

[15] S.L. Braunstein, S. Pirandola and K. Życzkowski, Better Late than Never: Information Retrieval from Black Holes, Phys. Rev. Lett. 110 (2013) 101301 [arXiv:0907.1190] [INSPIRE].

[16] S.D. Mathur, The Information paradox: A Pedagogical introduction, Class. Quant. Grav. 26 (2009) 224001 [arXiv: 0909.1038] [INSPIRE].

[17] V. Cardoso, E. Franzin and P. Pani, Is the gravitational-wave ringdown a probe of the event horizon?, Phys. Rev. Lett. 116 (2016) 171101 [arXiv: 1602.07309] [INSPIRE].

[18] V. Cardoso, S. Hopper, C.F.B. Macedo, C. Palenzuela and P. Pani, Gravitational-wave signatures of exotic compact objects and of quantum corrections at the horizon scale, Phys. Rev. D 94 (2016) 084031 [arXiv: 1608.08637] [INSPIRE].

[19] Virgo, Ligo Scientific collaboration, B.P. Abbott et al., Observation of Gravitational Waves from a Binary Black Hole Merger, Phys. Rev. Lett. 116 (2016) 061102 [arXiv: 1602.03837] [INSPIRE].

[20] J. Abedi, H. Dykaar and N. Afshordi, Echoes from the Abyss: Evidence for Planck-scale structure at black hole horizons, arXiv:1612.00266 [INSPIRE].

[21] G. Ashton et al., Comments on: "Echoes from the abyss: Evidence for Planck-scale structure at black hole horizons", arXiv:1612.05625 [INSPIRE].

[22] J. Abedi, H. Dykaar and N. Afshordi, Echoes from the Abyss: The Holiday Edition!, arXiv: 1701.03485 [INSPIRE].

[23] S.H. Shenker and D. Stanford, Black holes and the butterfly effect, JHEP 03 (2014) 067 [arXiv: 1306.0622] [INSPIRE].

[24] S.H. Shenker and D. Stanford, Stringy effects in scrambling, JHEP 05 (2015) 132 [arXiv: 1412.6087] [INSPIRE].

[25] J. Polchinski, Chaos in the black hole S-matrix, arXiv:1505.08108 [INSPIRE].

[26] J. Maldacena, S.H. Shenker and D. Stanford, A bound on chaos, JHEP 08 (2016) 106 [arXiv: 1503.01409] [INSPIRE].

[27] G. Turiaci and H. Verlinde, On CFT and Quantum Chaos, JHEP 12 (2016) 110 [arXiv: 1603.03020] [INSPIRE]. 
[28] K. Hashimoto and N. Tanahashi, Universality in Chaos of Particle Motion near Black Hole Horizon, Phys. Rev. D 95 (2017) 024007 [arXiv:1610.06070] [InSPIRE].

[29] E.T. Akhmedov, H. Godazgar and F.K. Popov, Hawking radiation and secularly growing loop corrections, Phys. Rev. D 93 (2016) 024029 [arXiv:1508.07500] [INSPIRE].

[30] A. Ashtekar, V. Taveras and M. Varadarajan, Information is Not Lost in the Evaporation of 2-dimensional Black Holes, Phys. Rev. Lett. 100 (2008) 211302 [arXiv:0801.1811] [INSPIRE].

[31] S.R. Das and S. Mukherji, Boundary dynamics in dilaton gravity, Mod. Phys. Lett. A 9 (1994) 3105 [hep-th/9407015] [INSPIRE].

[32] A. Almheiri and J. Sully, An Uneventful Horizon in Two Dimensions, JHEP 02 (2014) 108 [arXiv: 1307.8149] [INSPIRE].

[33] T.D. Chung and H.L. Verlinde, Dynamical moving mirrors and black holes, Nucl. Phys. B 418 (1994) 305 [hep-th/9311007] [INSPIRE].

[34] K. Schoutens, H.L. Verlinde and E.P. Verlinde, Black hole evaporation and quantum gravity, in Proceedings of Strings '93, Berkeley U.S.A. (1993), pg. 22, and in Proceedings of Workshop on String Theory, Gauge Theory and Quantum Gravity, Trieste Italy (1993), pg. 1 [hep-th/9401081] [INSPIRE].

[35] A. Almheiri and J. Polchinski, Models of AdS 2 backreaction and holography, JHEP 11 (2015) 014 [arXiv: 1402.6334] [INSPIRE].

[36] K. Jensen, Chaos in AdS 2 Holography, Phys. Rev. Lett. 117 (2016) 111601 [arXiv: 1605.06098] [INSPIRE].

[37] J. Maldacena, D. Stanford and Z. Yang, Conformal symmetry and its breaking in two dimensional Nearly Anti-de-Sitter space, PTEP 2016 (2016) 12C104 [arXiv:1606.01857] [INSPIRE].

[38] J. Engelsöy, T.G. Mertens and H. Verlinde, An investigation of AdS $S_{2}$ backreaction and holography, JHEP 07 (2016) 139 [arXiv:1606.03438] [INSPIRE].

[39] S.R. Das and S. Mukherji, Black hole formation and space-time fluctuations in two-dimensional dilaton gravity and complementarity, Phys. Rev. D 50 (1994) 930 [hep-th/9401102] [INSPIRE].

[40] A. Strominger and L. Thorlacius, Conformally invariant boundary conditions for dilaton gravity, Phys. Rev. D 50 (1994) 5177 [hep-th/9405084] [INSPIRE].

[41] S. Bose, L. Parker and Y. Peleg, Hawking radiation and unitary evolution, Phys. Rev. Lett. 76 (1996) 861 [gr-qc/9508027] [INSPIRE].

[42] S. Bose, L. Parker and Y. Peleg, Predictability and semiclassical approximation at the onset of black hole formation, Phys. Rev. D 54 (1996) 7490 [hep-th/9606152] [INSPIRE].

[43] J.G. Russo, L. Susskind and L. Thorlacius, Cosmic censorship in two-dimensional gravity, Phys. Rev. D 47 (1993) 533 [hep-th/9209012] [InSPIRE].

[44] E.P. Verlinde and H.L. Verlinde, A quantum S-matrix for two-dimensional black hole formation and evaporation, Nucl. Phys. B 406 (1993) 43 [hep-th/9302022] [INSPIRE].

[45] K. Schoutens, H.L. Verlinde and E.P. Verlinde, Quantum black hole evaporation, Phys. Rev. D 48 (1993) 2670 [hep-th/9304128] [INSPIRE]. 
[46] Y. Peleg, S. Bose and L. Parker, Choptuik scaling and quantum effects in $2-D$ dilaton gravity, Phys. Rev. D 55 (1997) 4525 [gr-qc/9608040] [inSPIRE].

[47] M. Gaudin, Diagonalization d'une classe d'hamiltoniens de spin, J. Phys. 37 (1976) 1087.

[48] B. Feigin, E. Frenkel and N. Reshetikhin, Gaudin model, Bethe ansatz and correlation functions at the critical level, Commun. Math. Phys. 166 (1994) 27 [hep-th/9402022] [INSPIRE].

[49] E. Frenkel, Affine algebras, Langlands duality and Bethe ansatz, q-alg/9506003 [INSPIRE].

[50] D. Grumiller and R. McNees, Thermodynamics of black holes in two (and higher) dimensions, JHEP 04 (2007) 074 [hep-th/0703230] [INSPIRE].

[51] E. Poisson, A Relativist's Toolkit. The Mathematics of Black-Hole Mechanics, Cambridge University Press, Cambridge U.K. (2004).

[52] J. Teschner, Quantization of the Hitchin moduli spaces, Liouville theory and the geometric Langlands correspondence I, Adv. Theor. Math. Phys. 15 (2011) 471 [arXiv:1005.2846] [INSPIRE].

[53] P. Di Francesco, P. Mathieu and D. Sénéchal, Conformal Field Theory, Springer-Verlag, New York U.S.A. (1997).

[54] S. Yu. Vernov, Construction of solutions for the generalized Henon-Heiles system with the help of the Painleve test, Theor. Math. Phys. 135 (2003) 792 [math-ph/0209063] [InSPIRE].

[55] S.J. van Tongeren, Introduction to the thermodynamic Bethe ansatz, arXiv:1606.02951 [INSPIRE].

[56] Y. Kiem and D. Park, Static and dynamic analysis of a massless scalar field coupled with a class of gravity theories, hep-th/9504021 [INSPIRE].

[57] M.W. Choptuik, Universality and scaling in gravitational collapse of a massless scalar field, Phys. Rev. Lett. 70 (1993) 9 [InSPIRE].

[58] C. Gundlach, Critical phenomena in gravitational collapse, Phys. Rept. 376 (2003) 339 [gr-qc/0210101] [INSPIRE].

[59] V.V. Bazhanov, S.L. Lukyanov and A.B. Zamolodchikov, Integrable structure of conformal field theory, quantum KdV theory and thermodynamic Bethe ansatz, Commun. Math. Phys. 177 (1996) 381 [hep-th/9412229] [INSPIRE].

[60] A. Ashtekar, F. Pretorius and F.M. Ramazanoglu, Surprises in the Evaporation of 2-Dimensional Black Holes, Phys. Rev. Lett. 106 (2011) 161303 [arXiv:1011.6442] [INSPIRE].

[61] A. Ashtekar, F. Pretorius and F.M. Ramazanoglu, Evaporation of 2-Dimensional Black Holes, Phys. Rev. D 83 (2011) 044040 [arXiv:1012.0077] [INSPIRE].

[62] D.G. Levkov, A.G. Panin and S.M. Sibiryakov, Unstable Semiclassical Trajectories in Tunneling, Phys. Rev. Lett. 99 (2007) 170407 [arXiv:0707.0433] [INSPIRE].

[63] F. Bezrukov, D. Levkov and S. Sibiryakov, Semiclassical S-matrix for black holes, JHEP 12 (2015) 002 [arXiv:1503.07181] [INSPIRE]. 